\title{
On Optimizing VLC Networks for Downlink Multi-User Transmission: A Survey
}

\author{
Mohanad Obeed, Student Member, IEEE, Anas M. Salhab, Senior Member, IEEE, \\ Mohamed-Slim Alouini, Fellow, IEEE and Salam A. Zummo, Senior Member, IEEE,
}

\begin{abstract}
The evolving explosion in high data rate services and applications will soon require the use of untapped, abundant unregulated spectrum of the visible light for communications to adequately meet the demands of the fifth-generation (5G) mobile technologies. Radio-frequency (RF) networks are proving to be scarce to cover the escalation in data rate services. Visible light communication (VLC) has emerged as a great potential solution, either in replacement of, or complement to, existing RF networks, to support the projected traffic demands. Despite the prolific advantages of VLC networks, VLC faces many challenges that must be resolved in the near future to achieve a full standardization and to be integrated to future wireless systems. Here, we review the new, emerging research in the field of VLC networks and lay out the challenges, technological solutions, and future work predictions. Specifically, we first review the VLC channel capacity derivation, discuss the performance metrics and the associated variables; the optimization of VLC networks are also discussed, including resources and power allocation techniques, user-to-access point (AP) association and APs-toclustered-users-association, APs coordination techniques, nonorthogonal multiple access (NOMA) VLC networks, simultaneous energy harvesting and information transmission using the visible light, and the security issue in VLC networks. Finally, we propose several open research problems to optimize the various VLC networks by maximizing either the sum rate, fairness, energy efficiency, secrecy rate, or harvested energy.
\end{abstract}

Index Terms-Visible light communication, hybrid VLC/RF networks, load balancing, non-orthogonal multiple access, energy harvesting, physical layer security.

\section{INTRODUCTION}

With the dramatic increase in total data traffic (approximately 7.24 exabyte-per-month in 2016, predicted to be 48.95 exabyte-per-month in 2021 [1]), there is an urgent need to develop a fifth-generation $(5 \mathrm{G})$ of networks with a higher system-level spectral efficiency that will offer higher data rates, massive device connectivity, higher energy efficiency (EE), lower traffic fees, a more robust security, and ultralow latency [2]-[4]. With the advent of the internet-ofthings (IoT) era, the amount of the connected devices to the internet is increasing dramatically [5], [6], resulting in a significant increase in data traffic that, and hence, crowded traditional radio-frequency $(\mathrm{RF})$ or wireless-fidelity (WiFi) networks [7]. Small cells or network densification have been

M. Obeed, A. M. Salhab, and S. A. Zummo are with the Department Electrical Engineering, King Fahd University of Petroleum \& Minerals (KFUPM), Dhahran, Eastern Province, Saudi Arabia (email: g201106250@kfupm.edu.sa, salhab@kfupm.edu.sa, zummo@kfupm.edu.sa).

M.-S. Alouini is with the Department Computer, Electrical, and Mathematical Siences \& Engineering, King Abdullah University of Science and Technology (KAUST), Thuwal, Makkah Province, Saudi Arabia (email: slim.alouini@kaust.edu.sa) proposed as a solution for 5G technologies [8], [9] in order to increase the system capacity and coverage, reduce the power consumption of mobile devices, and enhance the networks' EE. The continuity of dramatic growing in data traffic demand has motivated researchers to explore new spectrum, new techniques, and new network architectures to meet these demands. Visible light communication (VLC) has been introduced as a promising solution for $5 \mathrm{G}$ and beyond. The motivation behind emerging the VLC technology is the great invention of the energy-efficient light emitting diode (LED) [10]. White LEDs outperform the other light sources with their modulation performance, high electricalto-optical conversion efficiency, long life span, small size and light weight, low cost, and operational speed [11]-[13]. LED lamps consume approximately $20 \%$ of the power consumed by fluorescent bulbs and approximately $0.5 \%$ of the power consumed by traditional light sources [14].

Visible light communication uses a portion of the electromagnetic spectrum that is entirely untapped, free, safe, and provides a high potential bandwidth for wireless data transmission with rejecting the present RF interference [15]. Hence, VLC is a communication technology that uses LEDs as transmitters to emit both the light and information signals to the users. We should note that the power of the information signal must meet the illumination requirements, as well as being within the range of the LED's physical limits [16]. However, the non-linearity of LEDs in electrical-tooptical transfer can be efficiently avoided using pre-distortion mechanisms [17]. The VLC receiver contains a photo-detector (PD) component that has the ability to convert the received light intensity to a current signal. Data are transmitted using an intensity modulation (IM) technique at the transmitter, and received using a direct detection (DD) technique at the receiver (IM/DD) [18]. This means that the modulating signal must be real non-negative, and the existing modulation techniques in the RF networks adjusted to fit this property. Compared to RF networks, VLC networks provide higher data rates, larger EEs, lower battery consumption, and smaller latency. In addition, VLC can be safely used in sensitive environments such as chemical plants, aircraft, and hospitals [19]. Because of the small coverage of the transmitters in VLC systems, an exhaustive reuse of frequency can be implemented. VLC is also power-saving since the consumed power for communication is already used for illumination and may also be used for energy harvesting. Because the light can be blocked by opaque objects, VLC functions properly only in line-of-sight (LoS) communications, which own a robust security since the unauthorized users who are out of sight 
cannot receive an information signal of good quality.

Despite all the aforementioned VLC advantages, VLC faces many technical challenges that must be resolved in the near future to achieve its full standardization and integration with future wireless systems. The important challenges in VLC networks are: small bandwidth of LEDs, deriving the exact channel capacity, channel estimation and shadowing effects, backhauling VLC traffic into large-scale networks, the rapid decrease in light intensity with distance, and the noise or interference that may be generated by nearby lighting systems. One common solution to partially overcome these challenges is to optimize the parameters of VLC networks. Another common solution is to supplement the VLC networks by RF networks.

Numerous studies have investigated the potential applications of VLC to outdoor communications; yet, VLC is better suited for indoor communications. According to various published statistics, users of wireless information spend $80 \%$ and $20 \%$ of their time in indoor and outdoor environments, respectively [20]. In general, this paper reviews the optimization techniques studied in the literature to improve VLC systems' performance with focusing on target research directions.

\section{A. Related Work}

Several review articles have been written in the past on the topic of the VLC technology [21]-[31], but none of them addressed how the new emerging technologies in RF networks could be mapped and applied in VLC networks such as the non-orthogonal multiple access (NOMA), energy harvesting (EH), simultaneous wireless information and power transfer (SWIPT), space division multiple access (SDMA), and physical layer security (PLS). Specifically, Kumar et al. reviewed LED-based VLC systems and applications in their early stage development [21]. In [22], authors focused on the dual function of LEDs (used in smart lighting and VLC), and explored their potential for integration by introducing a new concept: LIGHTNETs (LIGHTing and NETworking) that performs both functions simultaneously. Authors of [23] highlighted the benefits and disadvantages of VLC networks, compared with RF networks. The benefits of LEDs for illumination and communications, modulation schemes, dimming techniques, and the methods used for improving the performance of VLC were reviewed in [24], while in [25], authors focused on the VLC link level transmission and shed some light on medium access techniques and visible light sensing. A more recent study by $\mathrm{Li}$ et al. reviewed system-level VLC networks, with a focus on user-centric network design, and compared it with the network-centric design with emphasizing on the interference reduction techniques [26]. In [27], authors explored the differences among optical wireless communications (OWC) technologies such as infrared communications, VLC, lightfidelity (LiFi), free space optical (FSO) communications, etc.

Some review articles focused on specific aspects of VLC such as VLC channel modeling methods [28], noise optical sources and noise mitigation mechanisms [29], VLC-based positioning techniques for indoor and outdoor applications [30], and the pertinent issues associated with the outdoor usage of VLC in vehicular communication [31]. They generally
TABLE I

LIST OF ABBREVIATIONS

\begin{tabular}{|c|c|}
\hline $4 \mathrm{G}$ & Fourth generation \\
\hline $5 \mathrm{G}$ & Fifth generation \\
\hline $\mathrm{AC}$ & Alternative current \\
\hline ACO-OFDM & Asymmetrically clipped optical OFDM \\
\hline AP & Access point \\
\hline APA & Access point assignment \\
\hline BER & Bit error rate \\
\hline CoMP & Coordinated multi-point \\
\hline CSI & Channel-state-information \\
\hline CSK & Color shift keying \\
\hline DD & Direct detection \\
\hline DC & Direct current \\
\hline DCO-OFDM & Direct current optical OFDM \\
\hline EE & Energy efficiency \\
\hline EGT & Evolutionary game theory \\
\hline EH & Energy harvesting \\
\hline FoV & Field-of-view \\
\hline FFR & Fractional frequency reuse \\
\hline FR & Frequency reuse \\
\hline GEE & Global energy efficiency \\
\hline IM & Intensity modulation \\
\hline IoT & Internet of things \\
\hline LB & Load balancing \\
\hline LED & Light emitting diode \\
\hline $\mathrm{LiFi}$ & Light fidelity \\
\hline LoS & Line of sight \\
\hline MIMO & Multiple input multiple output \\
\hline MINLP & Mixed-integer nonlinear programming \\
\hline MISO & Multiple input single output \\
\hline MRC & Maximum ration combining \\
\hline MPPM & Multipulse pulse position modulation \\
\hline NGDP & Normalized gain difference power allocation \\
\hline NOMA & Non-orthogonal multiple access \\
\hline OFDM & Orthogonal frequency division multiplexing \\
\hline OFDMA & Orthogonal division multiple access \\
\hline OMA & Orthogonal multiple access \\
\hline OOK & On-off keying \\
\hline OPPM & Overlapping pulse width modulation \\
\hline OWC & Optical wireless communication \\
\hline PD & Photo-detector \\
\hline PD-NOMA & Power domain NOMA \\
\hline PDMA & Pattern division multiple access \\
\hline PIN & Positive-intrinsic-negative \\
\hline PLC & Power line communication \\
\hline PLS & Physical layer security \\
\hline PPM & Pulse position modulation \\
\hline PPP & Poisson point process \\
\hline PWM & pulse width modulation \\
\hline QoS & Quality-of-service \\
\hline RA & Resource allocation \\
\hline $\mathrm{RF}$ & Radio frequency \\
\hline RGB & Red, green, and blue \\
\hline RLL & Run length limited \\
\hline SCMA & Sparse code multiple access \\
\hline SDMA & Space-division-multiple-access \\
\hline SIC & Successive interference cancelation \\
\hline SINR & signal-to-noise and interference ratio \\
\hline SISO & single-input-single-output \\
\hline SLIPT & $\begin{array}{l}\text { Simultaneous lightwave for information and } \\
\text { power transfer }\end{array}$ \\
\hline SNR & Signal-to-noise-ratio \\
\hline SWIPT & $\begin{array}{l}\text { Simultaneous wireless for information and } \\
\text { power transfer }\end{array}$ \\
\hline TDMA & Time division multiple access \\
\hline VLC & Visible light communication \\
\hline WiFi & Wireless-fidelity \\
\hline
\end{tabular}


identified emerging challenges and proposed future research directions.

This paper explores all the optimization techniques, previously reported in the literature, that aim at improving the VLC network performance. Emphasis is placed on how the new technologies, emerged in RF networks, mapped or used in VLC networks such as NOMA, SWIPT, cooperative transmission, SDMA, and physical layer security.

Specifically,

- This paper provides, in Section II, an overview of VLC technology, defines and discusses the objectives and constraints that must be taken into account when optimizing VLC networks. Special emphasis is placed on channel capacity derivations, and the unique properties of VLC. We also discuss the variables, parameters, and constraints having an impact on the performance of VLC networks.

- All optimization techniques are reviewed in Section III and IV, including power and resource allocation, usersto-APs association, cell formation, APs' cooperation, and multiple-input-multiple-output MIMO techniques that used for mitigating the disadvantages of VLC networks to improve performance. This important topic was previously investigated by $\mathrm{Li}$ et al. [26]. However, their study was focused on the difference between user-centric and network-centric cell formations, and the interference reduction techniques, whereas in this paper, we place our attention on the techniques, used in RF/VLC and in VLC standalone networks, that are aiming at alleviating the limitations in VLC networks. In other words, we show how to formulate optimization problems, what are the techniques used for solving these optimization problems, how the different objectives, limitations, constraints are evaluated, and how added RF APs can remove standalone VLC network limitations.

- By reviewing all the work conducted on NOMA-VLC systems in Section V, we show how such systems are different from NOMA-RF architectures.

- In Section VI, we survey the various energy harvesting techniques used in VLC networks and show how this added function (energy harvesting) affects the illumination and communication functions that are implemented simultaneously, using LEDs.

- The topic of physical layer security in VLC networks is also reviewed in Section VII, including the different techniques proposed to improve the secure VLC communications.

- In Section VIII, we outline some remaining challenges and open research areas in hybrid VLC/RF and standalone VLC networks, NOMA-VLC networks, energy harvesting in VLC systems, and securing VLC networks. We present several ideas, which have not been previously investigated or proposed, to improve the performance of the different types of VLC networks.

With this article, our goal is to present a clear, comprehensive picture of what has been accomplished so far, in the field of VLC networks, and to present future research directions. A list of abbreviation used in this paper is presented in Table I, and the different types of VLC networks that considered in this paper are shown in Fig. 1.

\section{Fundamentals of VLC Systems}

Because of its unique properties, a VLC channel is different from a RF or any other communication technology; its optical signal is modulated via the intensity of the signal, without carrying any information in phase or in frequency; the transmitted signal is positive and real, the optical power is proportional to the mean of the input power signal (not to the mean square of the signal amplitude); the transmitted peak power is constrained by the LEDs dynamic range and the illumination requirements.

\section{A. VLC Elements}

Every communication system must consist of a transmitter, channel, and receiver. Here, we discuss the characteristics of a VLC's transmitter and receiver.

1) VLC Transmitter: The LED lamp is the most appropriate transmitter used for both illumination and communication purposes (see Introduction for details). Each lamp usually consists of one or multiple LEDs driven by a circuit that controls the intensity of the brightness, using the the 'flowingin' current. The function of the driver circuit is to transmit the data by modifying the flowing-in current, which, in turn, modifies the light intensity. The flowing-in current must be within the LEDs dynamic range in order for the output (light intensity) to be linearly proportional to the input current. Because it shows the objects as they are without changing their real colors, the white color is commonly used for illumination and communication. Two common schemes are generally used in design white LEDs. One uses a blue LED with a yellow Phosphor layer [32], the other uses a combination of three LEDs (red, green, and blue) [33].

Because of its low cost and simplicity of implementation, the first type of LEDs (the blue LED with a yellow phosphor layer) is more popular than the RGB type for designing white LEDs. However, it has a limited bandwidth, compared to RGB, because of the slow absorption and emission of the coating phosphor layer. Khalid et al. [32] showed that a 1 Gbps data rate could be achieved, using this type of LEDs. The RGB technique is better for communication as it uses the color shift keying (CSK) modulation technique that modulates the signal, using the three different LEDs. By doing so, data rates of 3.4 Gbps data can be achieved [33].

One important issue that should be considered, when designing the VLC, is the illumination requirements, which is the main purpose of the LED. In other words, the illumination range that is required should not be violated by the VLC system. This means that the performance of the VLC system is related to the illumination design requirements (more details are given in Section II-D5).

2) VLC Receiver: The PD is a diode device sensitive to the light intensity that can convert the received light to a current modulated by the intensity of the light received. The PDs that are commercially available can easily take samples of the received visible light at a rate of tens of $\mathrm{MHz}$ [25].

There are three types of devices that can be used as VLC receivers of the optical signal coming from the LED 


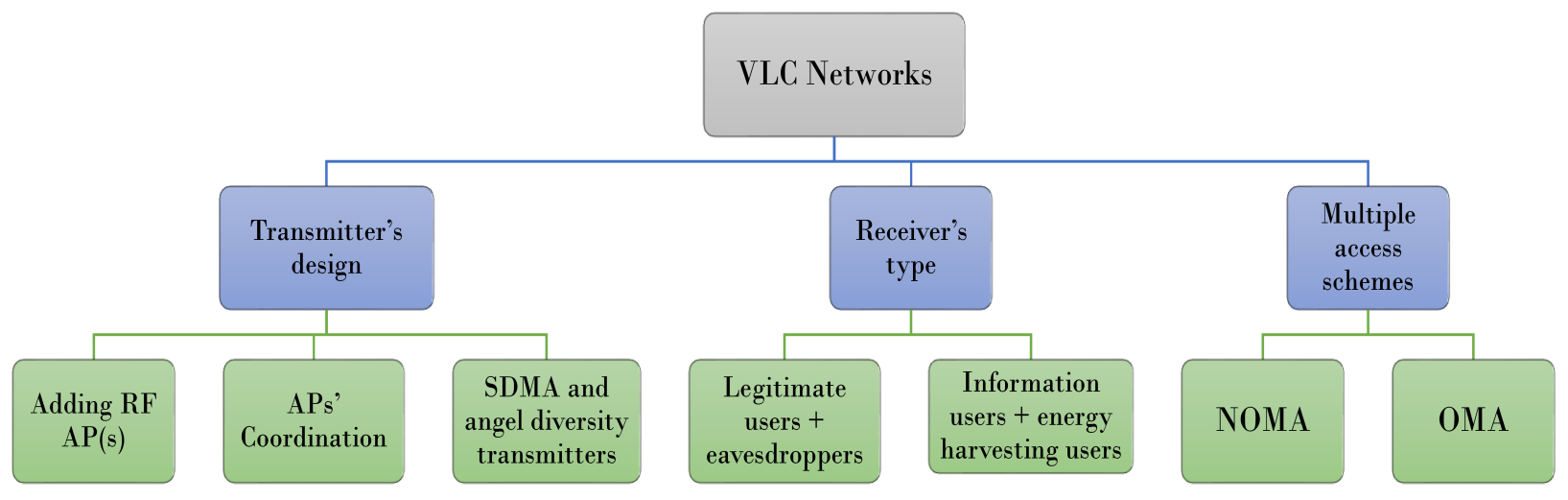

Fig. 1. Different types of VLC networks

transmitter: 1) photo-detector (e.g. positive-intrinsic-negative (PIN) and avalanche PD), 2) an imaging or camera sensor, 3) and a solar panel.

One of the advantages of a camera sensor is its availability on most mobile devices such as smart-phones used to capture videos and images. The main advantage of a solar panel is that it can directly convert the received light to an electrical signal without the need for an external power supply [34].

\section{B. Channel Model}

The receiver receives the LoS optical signal and many copies of non-LoS, coming from reflections. According to [35], the optical power received from signals reflected more than once is negligible. Fig 2 shows a channel model of VLC links, containing the LoS and first reflected links. The LoS VLC link between the AP $i$ and the user $j$ can be expressed as follows [13], [36]:

$$
h_{j, i}=\frac{A_{p}(m+1)}{2 \pi d_{j, i}^{2}} \cos ^{m}(\phi) g_{o f} \cos (\theta) f(\theta),
$$

where $A_{p}$ is the physical area of the receiver $\mathrm{PD}, m$ is the Lambertian index given by $m=\frac{-1}{\log _{2}\left(\cos \left(\theta_{1 / 2}\right)\right.}$, with $\theta_{1 / 2}$ the half-intensity radiation angle, $d_{j, i}$ the distance between $\mathrm{AP} i$ and user $j, g_{o f}$ the gain of the optical filter, $\phi$ the angle of irradiance at the AP, $\theta$ the angle of incidence at the PD, and $f(\theta)$ the optical concentrator gain is given by

$$
f(\theta)= \begin{cases}\frac{n^{2}}{\sin ^{2}(\Theta)}, & 0 \leq \theta \leq \Theta ; \\ 0, & \theta>\Theta,\end{cases}
$$

where $n$ is the refractive index and $\Theta$ is the semi-angle of the field-of-view (FoV) of PD. Komine and Nakagawa [35] showed that the DC attenuation of the channel, from the first reflected link is given by

$$
\begin{aligned}
d h_{1}=\frac{(m+1) A_{p}}{2 \pi d_{k, i}^{2} d_{j, k}^{2}} \rho d A_{s} \cos ^{m}\left(\phi_{r}\right) \cos \left(\alpha_{1}\right) \cos \left(\alpha_{2}\right) g_{o f} \\
\times f\left(\theta_{r}\right) \cos \left(\theta_{r}\right),
\end{aligned}
$$

where $\alpha_{r}$ and $\theta_{r}$ are the angels of the irradiance and incidence of the first reflection link, respectively, $d_{k, i}^{2}$ and $d_{j, k}^{2}$ are the distance from the AP $i$ to the reflecting point $k$ and the

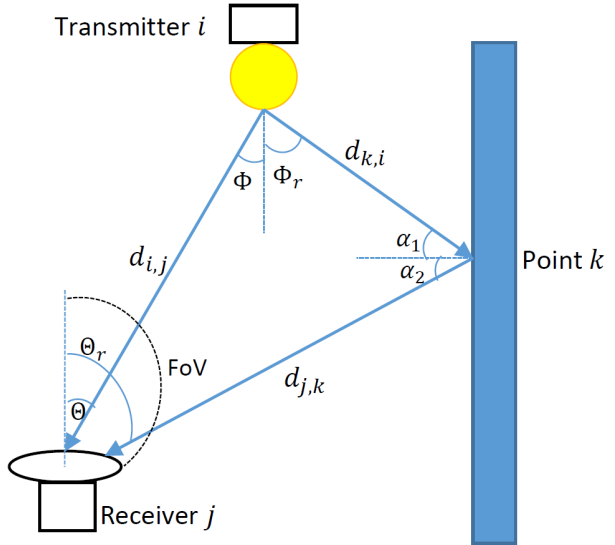

Fig. 2. Channel model, including the LoS link and the first reflected link

distance from the reflecting point $k$ to the user $j$, respectively, $\rho$ and $d A_{s}$ are the reflection factor and the reflective area, respectively, $\alpha_{1}$ and $\alpha_{2}$ are the irradiance angles with respect to the reflected point and with respect to the receiver, respectively.

The above model is the general description of the VLC channel model. However, for an accurate representation for the VLC channel model, practical channel characteristics should be considered. For more information about channel models, we refer the interested reader to [28] and [37]. However, these references did not cover the effect of the device orientation on the channel model and the MIMO channels. In the following, we summarize the effect of the device orientation on the channel model and show the MIMO channel characteristics.

1) Device Orientation Effect: Most of the existing studies in the literature assumed that all receiver devices are vertically upward for simplicity. However, users with smart-phones, which are the most common devices, tend to have their devices oriented in their preferable direction which is not necessary to be parallel with ground. This orientation information can be measured using a gyroscope that is equipped in smart-phones and then can be fedback to the transmitter to attain the channel information. In [38], Soltani et al. showed 
that the arbitrary users' orientation has a significant impact on the channel quality and load balancing. In [39], authors made an experiment for 40 participants to model the statistical distribution of the devices' random orientation and use it to characterize the VLC channel distribution. Eroglu et al. [40] characterized the LoS VLC channel when the incidence angle fluctuates randomly under any random distribution. They derived the square-channel statistics with deterministic and stochastic user's location and with wide and narrow user's FoV.

2) MIMO VLC Channel Model: In the literature, the channel in MIMO-VLC channel matrix is highly correlated since there is no phase or frequency information in IM/DD modulation scheme. However, having spaced LEDs reduces the channel matrix correlation and increases the reliability of decoding the parallel channels at the receivers [37]. In this case, the temporal delays that stem from the different distances between the different LEDs and receivers or stem from the different wire topologies should be considered. Authors of [41] showed that the difference in the distances between LEDs and the receiver results in a complex channel gain and phase differences in the frequency domain. Yesilkaya et al. [42] considered practical issues for accurately modeling the MIMO channel such as number of LED chips per luminary, spacing between LEDs, objects inside the room, and propagation delay of cables. These factors have impacts on the channel DC gain, root mean square (RMS) delay spread, and mean excess delay spread.

\section{VLC Modulation Schemes}

As mentioned previously, data cannot be transmitted by encoding the phase or frequency, and the modulation in VLC is implemented by varying the light intensity of the LED. On the other hand, the demodulation can be implemented by direct detection at the PD. Various IM/DD-based modulation techniques have been proposed and published in the literature. On-off keying (OOK) was proposed for VLC, as a simple modulation scheme, where data are represented by two levels of light intensity [43], [44]. In order to obtain higher data rates, in comparison with what OOK offers, pulse width modulation (PWM) and pulse position modulation (PPM) schemes, in which data are represented by the pulse width and the pulse position, respectively, have been proposed. The data rate in PWM can be increased by combining it with the discrete multitone technique (DMT) [45], while the data rate can be increased in PPM by using overlapping PPM (OPPM) [46], multi-pulse PPM (MPPM) [47], or the overlapping MPPM [48].

Due to the non-linear VLC channel response, the aforementioned modulation schemes suffer from inter-symbol interference. To combat this impairment, the orthogonal frequency division multiplexing (OFDM) scheme, widely used in RF systems, should be modified to be compatible with the IM/DD technique. Because the light signal is a real non-negative signal, the complex bipolar signals generated by OFDM must be represented by real positive signals in VLC. The solution can be implemented by relaxing the Hermitian symmetry constraint and convert the bipolar signal to a unipolar signal.
Two types of optical-OFDMs are widely used as VLC modulation schemes: a DC-biased optical OFDM (DCO-OFDM) and an asymmetrically-clipped optical OFDM (ACO-OFDM). In DCO-OFDM [49], [50], a positive direct current is added to make sure that the signal is non-negative, and all the subcarriers are modulated to maximize the spectral efficiency. On the other hand, in ACO-OFDM, only odd subcarriers are used to modulate the data [51], resulting in a symmetric time domain signal.

\section{Objectives and Constraints in VLC Networks}

In this section, we present the established objectives for the design or optimization of the VLC networks and discuss the associated constraints that must be achieved. Certainly, some of the unique characteristics of VLC technology have generated new challenges, different from those in RF networks. As a result, the techniques used in traditional RF networks cannot directly be applied to VLC networks.

1) System Capacity: Several issues (that do not exist in the RF systems) must be considered, when deriving the VLC channel capacity. These are: 1) dimming requirements, 2) peak optical intensity constraint, 3) illumination requirements and the LED dynamic range, 4) and necessity for the input signal to be non-negative and real-valued. In addition, the channel gain for VLC is modeled almost as the Lambertian model [35], in which the channel gain for VLC is timeinvariant but affected by geometrical parameters such as the locations of the transmitter and receiver. Because of the differences between RF and VLC systems, the capacity-achieving input distribution does not have to be Gaussian [52]. This means that the commonly derived Shannon channel capacity formula used for RF systems cannot be applied to VLC ones. Consequently, many researchers have been investigating the VLC channel capacity under these constraints. Several papers have focused on the optical intensity channel capacity, where only two constraints are imposed: the non-negative real-valued intensity signals, and the average and peak light intensity for eye safety standard [53]-[57].

In particular, the capacity of the scalar Gaussian channel constrained by amplitude power was proved to be achievable by a unique discrete random variable with a finite number of values [53]. For point-to-point transmission with peak and average power constraints, Chen et al. [54] showed that the capacity-achieving input distribution for the optical channel is discrete with a finite number of mass points. For multiple access channel, it was shown that the largest weighted sum-rate can be achieved by generating the codebooks of users according to discrete distributions with a limited number of mass points [55]. Authors in [56] showed that to obtain the capacity-achieving distribution, a finitedimensional optimization problem can be formulated, and efficient numerical algorithms can be proposed using standard optimization techniques to find the channel capacity. Authors of [57] showed that the capacity region of broadcast channel under non-negativity, peak, and average intensity constraints is achieved by superposition coding along with generating the codebooks of users according to discrete distributions with a finite number of mass points. 
Despite of all these studies which show that the optimal capacity-input distribution is discrete, a closed-form channel capacity is yet unknown. However, continuous input distributions provide a potential to have achievable rates that can be expressed in simple formulas, in contrast to discrete input distributions [58]. Hence, several papers focused on finding closed-form expressions for the upper and lower bounds of the channel capacity and on providing efficient approaches to tighten these bounds [58] [52], and [59]. A capacityapproaching distribution based on entropy maximization was developed in [58] aiming at reducing the complexity compared to the optimal input distribution. Lapidoth et al. [52] derived upper and lower bounds for the channel capacity and these bounds meet at low and high signal-to-noise-ratio (SNR) if the peak and average power constraints are imposed. Chaaban et al. [59] showed that the truncated-Gaussian input distribution can achieve higher rates than the distribution used in [52] in moderate SNR. In addition, authors of [59] provided a simple fitting functions for the achievable rate derived, numerically, in [58], which closely captures its achievable rate at any SNR.

For the VLC channel capacity, the illumination requirements and the LED dynamic range constraints must be considered when deriving the channel capacity. Ahn and Kwon [60] proposed a numerical approach to determine the channel capacity for inverse source coding in VLC, without providing a closed-form expression for the VLC channel capacity, whereas Wang et al. derived closed-form expressions for the upper and lower bounds of the dimmable VLC channel capacity [61]. The lower bound was expressed as follows:

$$
C \geq \frac{1}{2} \log _{2}\left(1+\frac{e}{2 \pi}\left(\frac{\zeta P}{\sigma}\right)^{2}\right),
$$

where $\zeta$ is the dimming target ranging from 0 to $1, P$ is the nominal optical intensity of LEDs, $\sigma^{2}$ is the Gaussian noise variance, and $e$ is the Euler parameter. The channel gain (losses and opto-electronic transformation factors) is assumed to be equal to 1, in Equation 4. Expression (4) is the common expression used in the literature to estimate the system capacity. The same authors derived closed-form expressions for the upper and lower bounds of the dimmable VLC channel, when imposing the peak optical intensity constraint of the LED [62]. This constraint resulted in a loss of channel capacity, and was found negligible when the maximum allowed optical intensity was twice the nominal optical intensity of the LEDs. With a different approximation method used for the intrinsic volumes of the simplex, Jiang et al. [63] derived a tighter upper bound, compared to that derived in [61], for the VLC dimmable channel capacity. Chaaban et al. derived the capacity bounds of the IM/DD optical broadcast channel under two constraints, which are the average light intensity and peak power intensity [64].

The channel in VLC system is often modeled as timeinvariant Lambertian model [35]. It does not change with time, but it changes with changing the location of the receivers. In other words, the VLC channel changes over the spatially geometrical locations [65]. Hence, authors of [65] studied the effect of user mobility on the channel capacity by assuming two different mobility patterns, which are the random walk and the main spot patterns. The authors derived a lower bound for the ergodic capacity and showed that the ergodic capacity decreases with the rise of the geometrical random variable. Another factor that affects the VLC channel capacity is the random receiver orientation. In [38], authors considered the impact of arbitrary receiver orientation on the system capacity. Their simulation results showed that the randomly rotated PDs degrade the user received services compared to the perpendicular PDs. Authors of [66] studied the effect of tilting the receiver angle on the channel capacity. The authors derived upper and lower bounds for the channel capacity and showed that the channel capacity can be improved by optimizing the tilt of the receivers angle.

Capacity of MIMO-VLC systems: For multiple-input-singleoutput (MISO) and MIMO optical intensity channel, authors of [67] and [68], respectively, found asymptotic capacity results for MISO and MIMO optical intensity channels in the high SNR case, while authors in [69] derived several upper and lower channel capacity bounds for the MISO optical intensity channel in low and high SNR regimes. For MIMO low-SNR regime, authors of [70] derived a general upper bound for the IM/DD channel capacity that was shown to be tight at low SNR regime. Using different approaches from [68], authors of [71] derived upper and lower bounds for high SNR-regime. In particular, authors of [68] used the entropy power inequality scheme which is suited for high-SNR, while authors of [71] used the QR-decomposition scheme to achieve higher rates at moderate SNR than the scheme in [68], when the number of transmitters antennas is less than or equal the number of equipped antennas at the receiver. For VLC channel with two users and two transmitters, Agarwal and Mohammed [72] proposed an achievable rate region of the proposed system VLC channel when the zero-forcing precoder was applied. They showed that the largest rate region was achieved when the average power of LED is half the maximum allowed peak power.

System Capacity with Secrecy Constraint: When VLC networks contain two types of users, the authorized users that have the authority to obtain and decode the data, and the eavesdroppers trying to obtain confidential messages without permission, the performance metric is changed to be the system capacity with secrecy constraint, which is called secrecy capacity. The secrecy capacity is defined as the maximum information rate that can be attained by the legitimate receiver minus the maximum eavesdropper's information rate [73]. Similar to the system capacity, the secrecy capacity with peak power constraint can be achieved by discrete input distributions with finite support [74]. The authors of [74] also showed that both the capacity and the secrecy capacity may not be achieved simultaneously. However, under sufficiently small peak power constraint, the capacity and the secrecy capacity can be both achieved by the symmetric binary distribution. For MISO case, authors of [75] used similar approach to the one in [74] with a non-straightforward modification to show that the capacity-achieving input distribution is discrete.

Different continuous input distributions, under intensity constraints, were proposed for seeking the simplicity and pro- 
viding closed-form expressions for upper and lower bounds of the secrecy capacity [76]-[80]. For the uniform input distribution and with considering amplitude constraint $|x(t)| \leq A \forall t$, the secrecy capacity of the single-input-single-output (SISO)VLC system is lower and upper bounded, respectively, by [81]

$$
C \geq \frac{1}{2} \log \frac{6 h_{D}^{2} A^{2}+3 \pi e \sigma^{2}}{\pi e h_{E}^{2} A^{2}+3 \pi e \sigma^{2}},
$$

and

$$
C \leq \frac{1}{2} \log \frac{h_{D}^{2} A^{2}+\sigma^{2}}{h_{E}^{2} A^{2}+\sigma^{2}},
$$

where $h_{D}$ is the transmitter-legitimate receiver channel, $h_{E}$ is the transmitter-eavesdropper channel, $\sigma^{2}$ is the noise variance, $e$ is the Euler parameter, and $A$ is the peak power constraint. It is important to note that the lower bound of the secrecy capacity must be greater than or equal zero, otherwise, a negative capacity would be obtained. This constraint can be achieved if the channel of the legitimate receiver $h_{D}$ is greater than the channel of the eavesdropper channel $h_{E}$. This constraint also guarantees that the upper bound is greater than the lower bound.

Authors of [77] used the truncated Gaussian inputs instead of the uniform input distributions for both the data and the artificial noise signals. In [79], authors used the truncated generalized normal distribution for the MISO-VLC Gaussian channel. It was shown that the truncated generalized normal distribution input performs better than the uniform and the Gaussian distribution inputs [79]. For the same system model and same input distribution as in [79], Arfaoui et al. [80] found a closed-form expression for the optimal beamforming, for a known location of the eavesdropper. Both the truncated Gaussian and the truncated generalized normal distributions provide potential to improve the secure communications by optimizing the parameters that cannot be optimized in the uniform distribution. Authors of [82] showed numerically that the discrete input distribution with a finite support significantly improves the secrecy rate, in MISO system, compared to those achieved by the truncated generalized normal and the uniform distributions. In [83], authors derived a closed-form for the achievable secrecy rate as a function of the discrete input distribution. They considered a class of discrete input distribution based on truncated discrete generalized normal distribution to enhance the achievable secrecy rate.

2) Throughput or Data Rate: This criterion is different from the system capacity because it calculates the actual transmitted data rate. Determining the bit error rate (BER) and the used coding and modulation schemes is required in finding the actual system throughput.

The data rate of the user $j$ can be expressed in OFDM systems using the following expression [84], [85]

$$
X_{j}=\frac{B}{\beta L} \sum_{i=1}^{\beta L-1} \eta_{i},
$$

where $B$ is the modulation bandwidth, $L$ is the number of subcarriers, $\eta_{i}$ is the $i$ th subcarrier spectral efficiency obtained from the modulation scheme, coding scheme, and the received signal-to-noise ratio (SNR) [86], and $\beta$ is a constant that depends on the kind OFDM used (e.g. for DCO-OFDM $\beta=\frac{1}{2}$, and for the traditional OFDM $\beta=1$ [85]). In DCO-OFDM, $L$ must be even to guarantee that half of the subcarriers are conjugates of the other half [84]. In TDMA systems, the achieved throughput, at the user $j$, is expressed as follows:

$$
X_{j}=\frac{B}{N_{T, j}} \sum_{i=1}^{N_{T, j}} \eta_{i},
$$

where $N_{T, j}$ is the number of time slots assigned for user $j$, and $\eta_{i}$ is the spectral efficiency of the $i$ th time slot.

3) Energy Efficiency: VLC networks are more energyefficient than RF networks because LEDs, used as transmitters, are power-saving devices, and the same power is used for both communication and illumination. Nevertheless, the transmitted power can be controlled within the given illumination limits to enhance the $\mathrm{EE}$. The $\mathrm{EE}$ is required to be improved for $5 \mathrm{G}$ wireless technologies to reduce the consumed power that is expected tremendous number of devices that will be connected. The EE can be improved by efficient resources optimization, power allocation, energy transfer and harvesting, and hardware solutions [87].

The common approach to guarantee energy-efficient systems is to optimally allocate the resources to maximize the EE function subject to QoS and maximum transmit power constraints. The EE function can be defined as the system's benefit over the total consumed power. In other words, if the system's benefit is the sum rate, then the EE is

$$
E E=\frac{R_{T}}{P_{T}},
$$

where $R_{T}$ is the sum rate and $P_{T}$ is the total consumed power at the transmitters.

Another way for improving the $\mathrm{EE}$ is to formulate the optimization problem as minimizing the total amount of transmitted power, under a given set of QoS constraints. This type of optimization problems is easier to tackle than the problem of maximization of the EE function. This is because the EE function is not concave, in terms of allocating the transmit power. The common approach to tackle the EE maximization problem is to convert the non-convex problem into a sequence of convex optimization problems using the Dinkelbach's method. Another way to improve the EE in VLC networks is to harvest the energy by converting the received light intensity into a current used for transmissions. This can be implemented by equipping the receivers with solar panels.

4) Fairness: Fairness is an important issue in VLC networks for many reasons: 1) the dramatic decrease in the VLC channel value with the distance between the transmitter and receiver makes many users unable to switch from crowded cells to uncrowded ones; 2) the small coverage stimulates designers to fully re-use the frequency in the cells, resulting in severe interference with the signal received by some users.

Fairness is commonly measured using Jain's formula [88] for a single cell or for the whole cellular system. The fairness 
among users in the $i$ th cell is given by

$$
F_{i}=\frac{\left(\sum_{j=1}^{N_{i}} R_{j, i}\right)^{2}}{N_{i} \sum_{j=1}^{N_{i}} R_{j, i}^{2}},
$$

while the fairness among users in $N_{a p}$ cells is given by

$$
F_{s}=\frac{\left(\sum_{i=1}^{N_{a p}} \sum_{j=1}^{N_{i}} R_{j, i}\right)^{2}}{N_{T} \sum_{i=1}^{N_{a p}} \sum_{j=1}^{N_{i}} R_{j, i}^{2}},
$$

where $N_{i}, N_{a p}$, and $N_{T}$ are the number of users associated with the cell $i$, the number of cells, and the total number of users in the system, respectively, and $R_{j, i}$ is the $j$ th user data rate associated with the cell $i$.

Fairness can be achieved either by formulating the optimization problem to maximize the utility with a guarantee to achieve a proportional fairness [89], $\alpha$-proportional fairness [90], or by adding the QoS constraints to the formulated optimization problem. The concept of the proportional fairness is to modify the objective function to imply both the system utility (e.g. sum-rate) and the fairness. If we denote $x$ as the utility function, the generalized objective function can be written as follows:

$$
\Gamma(x)= \begin{cases}\log (x), & \alpha=1 \\ \frac{x^{1-\alpha}}{1-\alpha}, & \alpha \geq 0, \alpha \neq 1,\end{cases}
$$

where $\alpha$ is a proportion factor. $\alpha=0$ means the utility is only considered and the fairness is ignored; $\alpha=1$ means that the proportional fairness is achieved, and, if $\alpha \rightarrow \infty$, the max-min fairness is achieved.

5) Required Illumination Constraints: The two functions of LED, illumination and communication, are related to each other and must be studied and optimized jointly. In other words, the illumination requirements should be considered in designing the input current to the transmitter LED. This requirement implies that different constraints must be considered when optimizing the communication in VLC networks. The constraints are the peak optical power, dimming requirements, and flicker reduction.

For the peak power constraint, we should note that the input signal to the LED contains two components: the alternative signal (that contains the information), and the DC signal used to guarantee non-negative signal. The total energy emitted by the LED determines the transmitted optical power and the subsequent received signal strength, whereas the brightness is determined by the luminous intensity [35]. We denote $\Phi_{\max }, \Phi_{\min }$, and $\Phi_{\text {avg }}$, as the predefined minimum illumination, maximum illumination, and the average illumination over the entire area, respectively. For the office work, an illuminance between 300 to 2500 lux is required [35].

The relation between the radiated optical power at LED and the luminous flux at the point $i$, which is distant from LED by $d_{i} \mathrm{~m}$ with incidence and radiance angles $\theta$ and $\psi$, respectively, can be given by [91], [92]

$$
h_{i} P_{\text {opt }}=\delta \Phi_{i},
$$

where $\delta$ is the optical to luminous flux conversion factor [92] which its value depends on the LED type; $P_{o p t}$ is the optical power, $\Phi_{i}$ is the luminous flux at point $i$, and $h_{i}$ is given by:

$$
h_{i}=\frac{m+1}{2 \pi d_{i}^{2}} \cos ^{m}(\theta) \cos (\psi),
$$

where $m$ is the Lambertian index given in Section II-B.

One additional constraint for communication is that the input DC-biased current (DC and AC currents) to the LED must be within the dynamic range of the LED to have the radiated optical power linearly proportional to the input current [93]. For instance, the practical dynamic range of the LED Vishy TSHG8200 is within [ $5 \mathrm{~mW}, 50 \mathrm{~mW}$ ].

To meet the illumination requirements at all points in the floor area, the upper and lower bounds of the optical power should be set accordingly. Considering both the bounds of the LED dynamic range and the illumination limits, the optical power at the transmitting LED must be confined by

$$
\max \left(P_{\min , i l l}, P_{\min , D}\right) \leq P_{\text {opt }} \leq \min \left(P_{\max , i l l}, P_{\max , D}\right),
$$

where $P_{m i n, i l l}$ and $P_{m a x}$,ill are the minimum and maximum optical power required for achieving the corresponding illumination requirements, respectively; $P_{\min , D}$ and $P_{\max , D}$ are the maximum and minimum power limits for the LED dynamic range, respectively.

The dimming control is a desirable process for the illumination purpose [94]. For power saving, LEDs can be dimmed to desired levels, using appropriate modulation schemes [43], such as multi-pulse position modulation (M-PPM) [95]; or variable OOK [96].

Another purpose for the used modulation scheme is to mitigate the light intensity fluctuation to be unnoticeable by the human eyes. To guarantee the flickering is above the human eyes' fusion frequency, flickering frequency must be at least greater than $200 \mathrm{~Hz}$ [97]; this can be avoided by using the Run Length Limited (RLL) codes that are used to reduce the long runs of $0 \mathrm{~s}$ and $1 \mathrm{~s}$.

6) Coverage Probability: Since the LEDs in VLC can cover only a small area, and the coverage probability decreases dramatically as the distance increases, the coverage is an important issue in VLC networks and should be considered when designing the networks' parameters. The coverage probability can be defined as the probability that the received data rate for typical user is greater than or equal to a certain data rate threshold. All the geometrical parameters of the VLC channel affect the coverage probability, but we focus our discussion on those having major impacts such as the distance, optical power intensity, and the user's field-of-view (FoV). If we consider a system model consisting of multiple VLC APs and the considered user $j$ is served only by one AP $i$, increasing the optical power would surely enhance the channel link from the AP $i$ to the user $j$, but would increase the interference from all other APs significantly. The user's FoV plays a significant role in affecting the coverage probability, since decreasing the user's FoV leads to enhancing the VLC channel and decreasing the number of interfering APs, but we should also note that an extensive decrease in the user's FoV leads to decrease of the coverage probability. On the other hand, for a given FoV, increasing the height of the APs leads to an increase in the number of APs in the user's field 


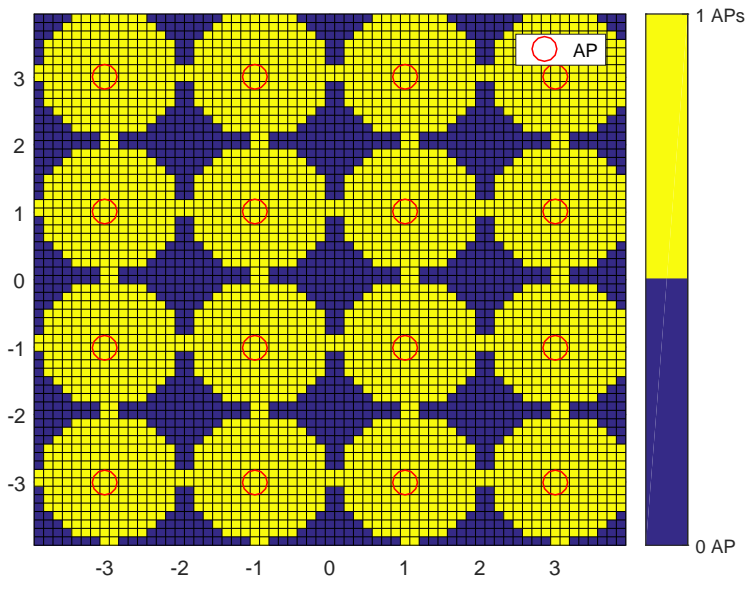

(a) $\mathrm{FoV}=30^{\circ}$

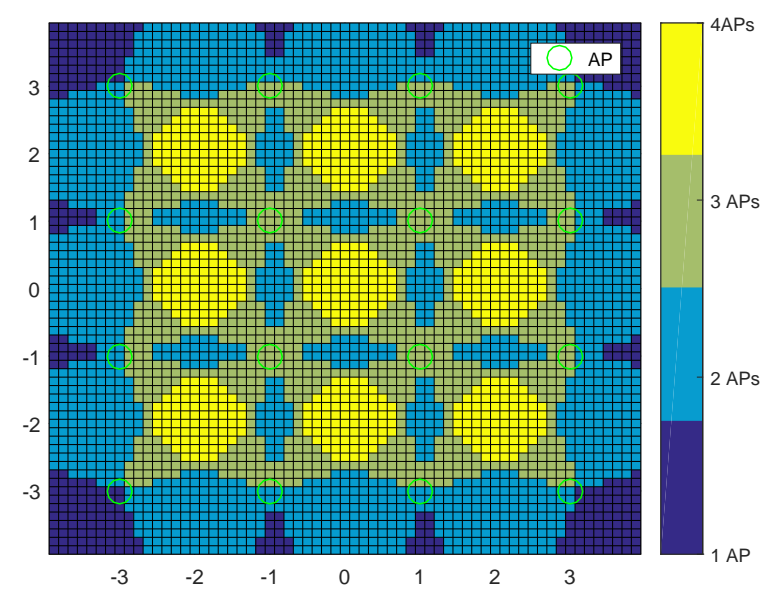

(c) $\mathrm{FoV}=50^{\circ}$

Fig. 3. Number of APs that can cover the area based on the user's FoV.

of view, meaning that the number of interfering APs would increase, and the path loss from the AP $i$ to the user would also increase.

Fig. 3 represents the effect of a user's FoV on the coverage probability by showing the number of APs that can cover the area with different user's FoV. Consider an $8 \times 8 \times 3$ $\mathrm{m}^{3}$ room equipped with $4 \times 4$ VLC APs that are at ceiling level and serving users with height $0.85 \mathrm{~m}$. In Fig. 3, we use the simulation parameters provided in Table II (for the information users) to find the LoS channel (1) form all APs to each point in the room with assuming that each point is a receiver with the given FoV. We adopt only the LoS link because it is dominating in VLC and the channel is degraded more than 90 percent if the LoS link is absent [98].

Fig. 3 shows that the coverage probability increases as the user's FoV increases. It shows the number of APs that can cover each spot in the room with the given FoV. On the other hand, Fig. 4 shows that the channel quality decreases as the user's FoV increases. In Fig. 4, we assume that we only have one AP fixed at the ceiling center and one receiver located

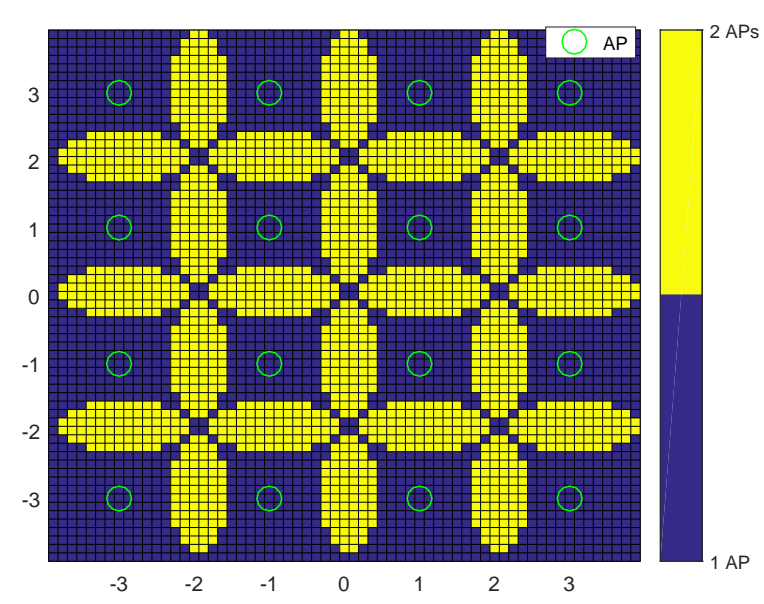

(b) $\mathrm{FoV}=40^{\circ}$

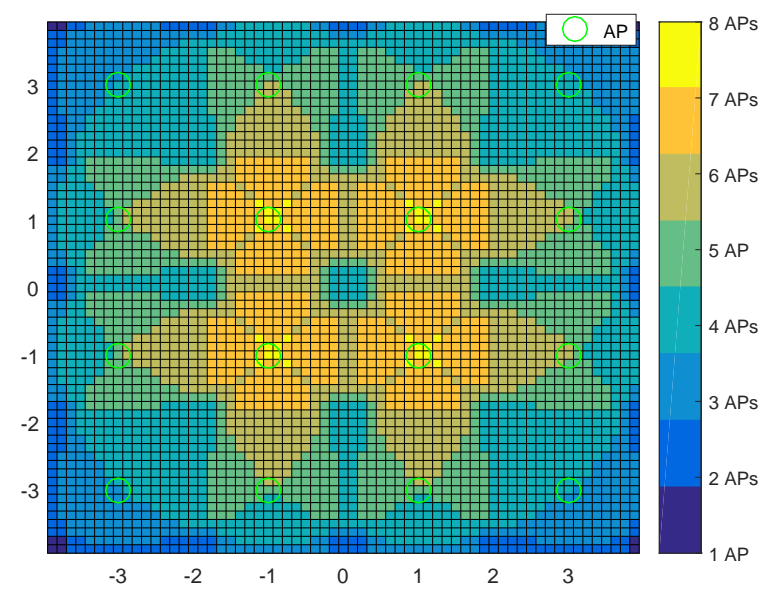

(d) $\mathrm{FoV}=60^{\circ}$ directly under the AP with the given distance and given FoV. We also use Table II to find the LoS channel (1). Figs. 3 and 4 show that the user's FoV has a great impact on the channel quality and the coverage probability, meaning that optimizing the FoV would have a significant impact on the VLC systems.

7) The Harvested Energy: An additional function to LEDs, besides the illumination and communication, is the transfer of power, using the light intensity. When the VLC network consists of users that need to harvest the energy, the parameters should be designed to find a compromise between the three functions. The receiver can harvest the energy by equipping it with a solar panel that can convert the received modulated light signal into an electrical signal without an external power supply. Because the received current signal at the receiver contains both DC and AC currents, the DC current can be blocked and forwarded to the energy harvesting circuit. Li et al., in [99], derived the energy that can be harvested by a user from one LED as:

$$
E=f I_{D C} V_{o c},
$$


TABLE II

SIMUlation PARAMETERS

\begin{tabular}{|l|l|}
\hline Name of the Parameter & Value of the Parameter \\
\hline Maximum bandwidth of VLC AP, $B$ & $20 \mathrm{MHz}$ \\
The physical area of a PD for information & $0.1 \mathrm{~cm}^{2}$ \\
users, $A_{p}$ & \\
The physical area of a PD for EH users, & $0.04 \mathrm{~m}^{2}$ \\
$A_{p}$ & \\
Half-intensity radiation angle, $\theta_{1 / 2}$ & $60^{\circ}$ \\
FoV semi-angle of PD, $\Theta$ & $30^{\circ}-60^{\circ}$ \\
Gain of optical filter, $g_{\text {of }}$ & 1 \\
Refractive index, $n$ & 1.5 \\
Efficiency of converting optical to electric, & 0.53 [A/W] \\
$\rho$ & \\
Maximum input bias current, $I_{H}$ & $12 \mathrm{~mA}$ \\
Minimum input bias current, $I_{L}$ & $0 \mathrm{~A}$ \\
Fill factor, $f$ & 0.75 \\
LEDs' power, $P_{\text {opt }}$ & $10 \mathrm{~W} / \mathrm{A}$ \\
Thermal voltage, $V_{t}$ & $25 \mathrm{mV}$ \\
Dark saturation current of the PD, $I_{0}$ & $10^{-10} \mathrm{~A}$ \\
Noise power spectral density of LiFi, $N_{0}$ & $10^{-21} \mathrm{~A} / \mathrm{Hz}$ \\
Room size, & $8 \times 8$ \\
Room height, & $2.5 \mathrm{~m}$ \\
User height & 0.85 \\
Number of VLC APs, & $4 \times 4$ \\
Number of users, & $5-35$ \\
Monte-Carlo for user distribution, & $100 \mathrm{different}$ user dis- \\
& tributions \\
\hline RF & 1 \\
\hline Number of RF APs & $(0,0) \mathrm{in}$ the ceiling \\
Location of RF AP & $10 \mathrm{Watt}$ \\
Transmit power & $5 \mathrm{~m}$ \\
The distance of breakpoint & $2.4 \mathrm{GHz}$ \\
Central carrier frequency & $20 \mathrm{MHz}$ \\
Bandwidth & $45^{\circ}$ \\
Angle of arrival/departure of LoS & $3 \mathrm{~dB}$ \\
Standard deviation of shadow fading (be- \\
fore the breakpoint) & \\
Standard deviation of shadow fading (after & $5 \mathrm{~dB}$ \\
the breakpoint) & $-174 \mathrm{dBm} / \mathrm{Hz}$ \\
Noise power spectral density & \\
& \\
\hline
\end{tabular}

where $f$ is a fill factor of approximately $0.75, I_{D C}$ the received DC current, and

$$
V_{o c}=V_{t} \ln \left(1+\frac{I_{D C}}{I_{0}}\right),
$$

where $V_{t}$ is the thermal voltage, and $I_{0}$ the dark saturation current of the PD. If we denote the transmitted DC current by $b$, the received DC current can be expressed as by $I_{D C}=\rho h P_{o p t}$. Hence, if we have multiple LEDs, the harvested energy at the user $j$ is given by:

$$
E_{j}=f \rho P_{o p t} V_{t} \mathbf{h}_{j}^{T} \mathbf{b} \ln \left(1+\frac{\rho \mathbf{h}_{j}^{T} P_{o p t} \mathbf{b}}{I_{0}}\right),
$$

where $\mathbf{h}_{j}$ is the channel vector between the LEDs and the user $j$, and $\mathbf{b}$ is the DC Bias current vector at LEDs.

\section{Resource AND POWER CONTROL With AP ASSIGNMENT}

In this section, we review the optimization techniques previously reported in the literature to improve the VLC network performance when the system consists of multi-users. Four main issues are considered in this type of networks, for maximizing the various objectives and achieving the various constraints discussed in Section II. These are: the user-to-network association (called 'access point assignment'

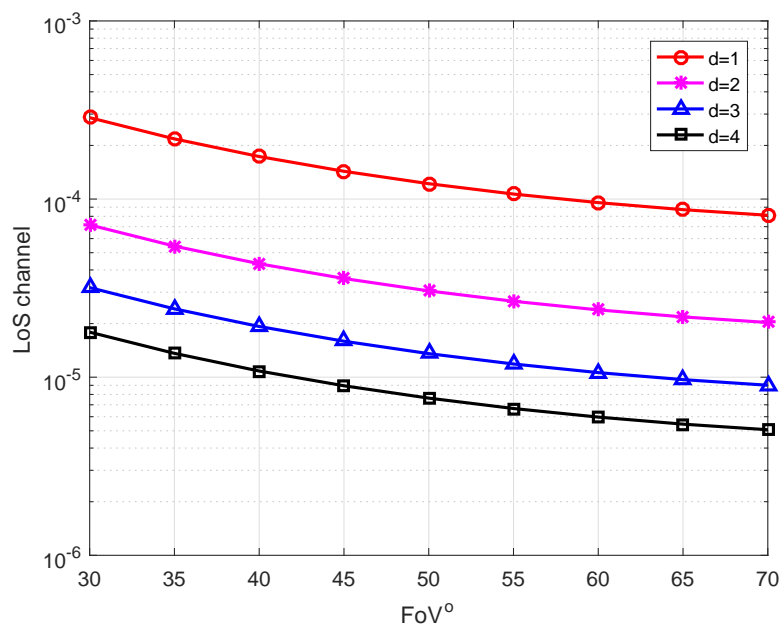

Fig. 4. The effect of user's FoV on the channel quality with different transmitter-receiver distance, when the angels of radiance and incidence are zero.

(APS)), resource management, power allocation, and APs coordination. The joint of APA and resource allocation was identified by load balancing (LB). LB has been extensively investigated in RF networks [100], [101]. However, the unique properties of VLC technology make the problem different, and the techniques used in RF networks cannot be directly applied to VLC networks.

Despite all the advantages of VLC systems mentioned in the Introduction, they suffer from several limitations that contribute to the degradation of the system's performance such as a small coverage area, non-LoS failure transmission, frequent handover, and inter-cell interference. This leads to unbalanced systems, with some users receiving a poor service, while others may receive a high QoS. In particular, the objects existed in rooms might block the LoS link of some intended receivers, leading to a degradation of the channel by up to 90 percent of the LoS channel [98], and, as a consequence, a significant deterioration of the data rates for the intended users. However, these opaque objects can block the intercell interference coming from the adjacent VLC APs for other users. This means that the fluctuation of the received QoS at users is high and that the blockages significantly affect the system fairness and the balance of the systems. Another cause for unbalanced VLC systems is the handover. For the reason that the coverage area of LEDs is small, the mobile users would suffer from wasting resources by sending and transmitting the overhead of the required handover. Fig. 5 shows how the handover is an important issue in VLC systems. As observed in the figure, the small coverage area of the LEDs in VLC networks leads to a decrease in the throughput of both the system and the mobile users due to the overhead generated by such handovers [102]-[104]. However, by dividing the time into sufficiently short periods, we can have quasi-static periods known as 'states'. The handover consumes time, on average from $30 \mathrm{~ms}$ to $300 \mathrm{~ms}$ [105]. Another issue due to the small coverage area is the fact that the crowded static users cannot be distributed to the 


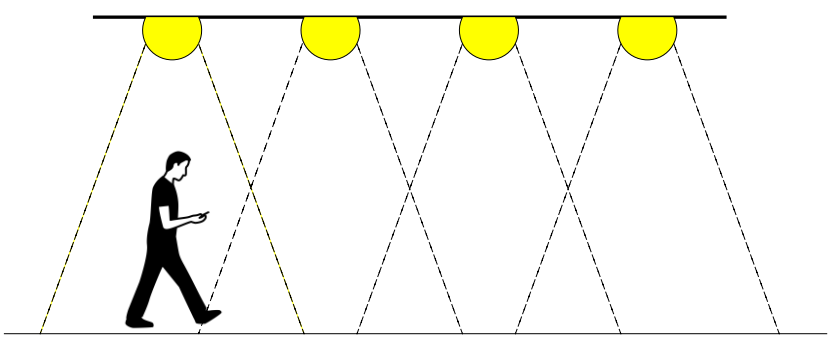

Fig. 5. Handover in VLC network.

deployed cells, resulting all or most of them will be connected to one cell. This causes some APs to be overloaded, and consequently leads to a poor service for the connected users, while the other APs are unloaded or have a lower number of users. The bright side of the VLC's small coverage area is the fact that the whole bandwidth can be fully re-used in all cells, which improves the spectral efficiency of the overall system [106]. However, re-using the full frequency in cells generates inter-cell interference, to some extent. Intercell interference can be accepted for the sake of improving the system's spectral efficiency. On the other hand, the services received by the users located at the edges of the cells would be affected by this inter-cell interference. To summarize, because of these issues, the users located at the edges of cells, blocked by objects, in motion, or connected to overloaded APs can not receive a good QoS like the other users. This significantly deteriorates both the performance and fairness of the VLC systems.

\section{A. Optimizing Hybrid VLC/RF Networks}

One of the most common solutions to the aforementioned VLC issues is to supplement the standalone VLC networks with RF networks. Compared to VLC networks, RF networks are known for their ubiquitous presence (high coverage area) and proper operation in non-LoS environments. In addition, the devices connected to RF networks do not suffer from VLC interference and vice-versa [107]. Therefore, adding one or more RF APs to VLC networks mitigates the LoS blockages, handover overhead, and inter-cell interference. However, a problem remains: finding a compromise between the high coverage area RF networks and the high capacity VLC networks. In other words, how to distribute the users among the APs (either RF or VLC) to improve the overall system's performance with an acceptable fairness of the system. The main idea is to associate the users who suffer from interference, handover overhead, and blockages to the RF AP(s) and keep the other users connected to the VLC networks. As shown in Fig. 3, when the users' FoV is $30^{\circ}$, the problem in VLC networks is the coverage, whereas, if the users' $\mathrm{FoV}$ is greater than or equal to $40^{\circ}$, the problem is the interference. In Figs. 6 and 7, we show how adding one RF AP to the VLC network can enhance the sum rate and system's fairness, respectively. We consider the same system model considered in Fig. 3 (the same number of APs and room dimensions). We also assume that the users are distributed uniformly in the floor with height $0.85 \mathrm{~m}$. Each result point is averaged over 150 different users' distributions in the room.
The RF AP is assumed to be located at the ceiling center of the room and the RF channel is modeled as in [108]. In these figures, we associate the uncovered users (when $\mathrm{FoV}=30^{\circ}$ ) and the interfered users (when $\mathrm{FoV}=40^{\circ}$ ) to the RF AP, while keeping the other users connected to the VLC network (each user is connected to the closest VLC AP). It can be seen from these figures that the system sum rate and fairness are improved significantly by adding one RF AP when the users' $\mathrm{FoV}$ is $30^{\circ}$ or $40^{\circ}$.

Several techniques have been proposed to balance the load and tackle these issues by an efficient user distribution among VLC/RF APs [85], [102]-[104], [108]-[120]. LB consists of two missions: the APs' assignment (APA) and allocating the resources, whether this resource is a time slot in TDMA schemes or a sub-carrier in OFDMA schemes. Specifically, Stefan and Haas [109] started to study the APA by distributing the users between one RF AP and one VLC AP. Some of the users were associated to the VLC AP to alleviate the load of the RF AP, and the infeasible VLC connections were transferred to the RF AP. In [110], by having multiple VLC and RF APs, the advantages of combining RF and VLC networks were investigated, and it was proposing that users can be distributed dynamically, on both the VLC and RF networks, based on the users' channel condition. Users can then migrate to the AP offering higher data rates. The APA was implemented in [110] under the assumption that the resources are allocated fairly among users. It was concluded that the hybrid VLC/RF networks improved the performance significantly, compared to the VLC or RF standalone networks. Authors of [104] proposed to first associate the users to the VLC network, and then, to re-allocate the users receiving a lower data rate than a predefined threshold to RF APs. In [111], authors formulated a centralized and distributed optimization problem for user association to the APs (whether this AP is VLC or RF AP) with allocating the resources jointly among users. The centralized optimization problem, with considering the proportional fairness [121], was formulated as a mixed- integer non-linear programming (MINLP), which is highly complex. Hence, a distributed algorithm was also proposed with lower complexity compared to the centralized algorithm.

To decrease the number of handovers, Wang and Haas [102] proposed a dynamic LB scheme in which the quasistatic users are connected to VLC APs, and the moving users are connected to the RF AP. In [112], [113], authors upgraded the formulated optimization problem in [111] to consider the handover in the dynamic systems. With considering the handover overhead and $\alpha$-proportional fairness, the authors of [112] formulated and proposed two solutions for two optimization problems, i.e. the joint APA and resource allocation problem (JOA), and the separate APA and resource allocation (SOA). They compared the two approaches in terms of performance and complexity. The former approach was found to achieve a better QoS for the users, but with a significant higher complexity, up to 1000 times greater, than the later. In a separate study [113], instead of assigning the users to a specific $\mathrm{AP}, \mathrm{Wu}$ et al. formulated the problem by considering the handover as a hierarchal assignment to first 
assign the network (either RF or VLC) to each user, and then select the appropriate AP, in the assigned network, for each user. Because the problem formulated in [111] is for static systems, those presented in [112], [113] provide a significant improvement in the system performance for dynamic systems.

Instead of considering the handover with $\mathrm{LB}, \mathrm{Wu}$ and Haas [114] considered the LoS VLC channel blockages in the formulated optimization problem. They modified the formulated optimization problem to accommodate the LoS VLC channel blockages. The main idea is that, the users that suffer from a high occurrence rate of channel blockages should travel to the RF networks, whereas the users that do not suffer from blockages, or the ones that suffer from a low rate of blockages (to avoid the effect of handover overhead), should stay in the LiFi networks.

To avoid the complexity of solving these optimization problems, fuzzy logic-based approaches were proposed for balancing the load in VLC networks [108], [115], and [116]. Authors of [115] and [108] proposed two-stage assignment process for the users in one RF AP and multiple VLC APs. They first decided which users should be connected to the RF AP, then they distributed the remaining users to the VLC APs, regardless of the presence of the RF AP and its connected users. In the fuzzy logic approach, the user $j$ scores the APs, based on its offered throughput, SNR, inter-cell interference from the adjacent APs, and activity of the adjacent VLC APs, then decides whether to connect to the RF AP or to the VLC network, based on the resulting score. Similarly, authors of [116] used this approach to handle the handover in a dynamic hybrid VLC/RF system model. In their scheme, they considered several parameters as an input to the fuzzy logic approach: the instantaneous and average CSI, user speed, and the minimum required data rate at users.

In [85], authors used another approach called the 'evolutionary game theory' (EGT), to solve the joint LB and resource allocation problem. Some practical issues were considered in their study, including the receiver's orientation angle, LoS blockage in RF and VLC APs, and the diversity in the users' data requirements. In addition, the channel of LiFi was characterized with considering these practical factors. Authors in [117] studied and compared the common approaches used for balancing the load in the hybrid VLC/RF networks which are: 1) optimization based algorithms, 2) evolutional game theory, 3) fuzzy logic based algorithms. They showed that, for the dynamic systems when the handover is considered besides the AP assignment and the resource allocation, the fuzzylogic-based algorithms outperformed the other approaches, whereas for the static systems, the optimization-based algorithms are the best, with a slight improvement over the simpler EGT approach.

Authors of [118] used a different approach for assigning the APs in the dynamic systems, using bandit theory with considering the accumulated reward gap function as a performance metric. Their idea was to consider the learning aided AP assignment that enables the system to adjust the AP selection probability depending on the learning historical reward information and the environmental information. In [103], authors distributed users to the APs by applying the

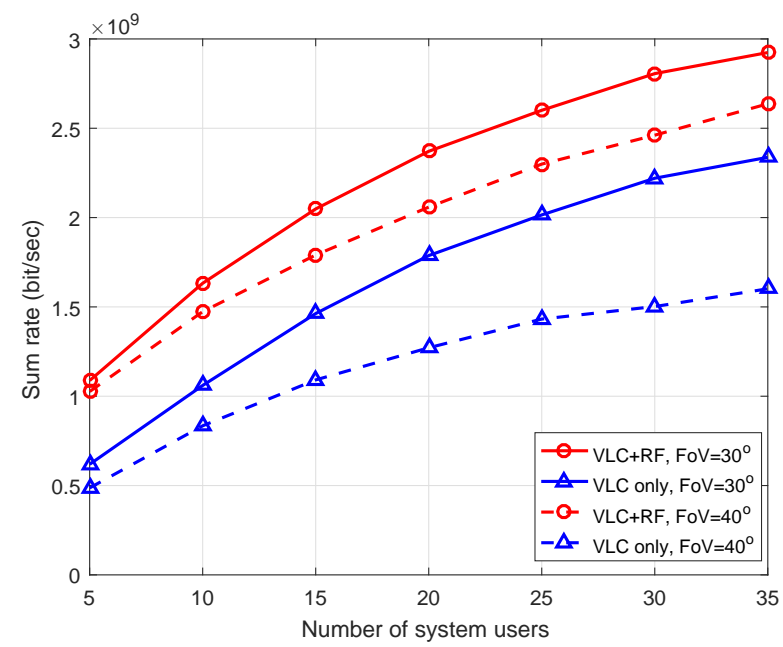

Fig. 6. Comparison of VLC/RF system and VLC alone by plotting the sum rate versus the number of system users with different users' FoV.

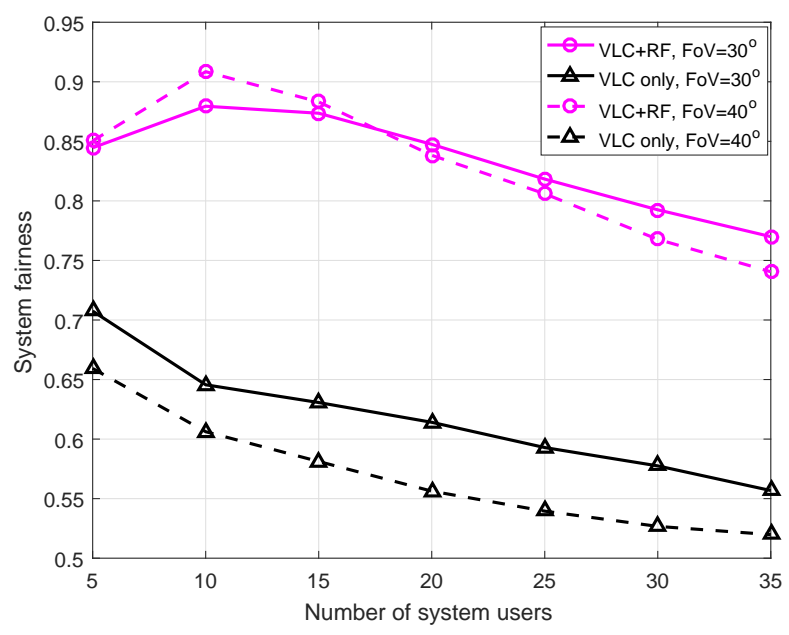

Fig. 7. Comparison of VLC/RF system and VLC standalone by plotting the system fairness versus the number of system users with different users' FoV.

matching theory, as the users were mapped to be students and the APs were mapped to be collages. Then, taking into account the preferences (i.e. system throughput, users' moving directions, and fairness index), students (users) would decide which collage (AP) is the best for them to maximize their preferences, in return, collages accept the maximum number of applicants to maximize their preferences, while rejecting the others. The rejected students would go to their second preferable collage (AP), and so on.

In a different way, we proposed new algorithms for joint APA and power allocation aiming to improve both the system capacity and fairness [119], [120]. Because the assignment of APs, power allocation, and determination of the exact interference information are interlinked problems, iterative algorithms were proposed to efficiently jointly distribute the users to APs, and to distribute the powers of the APs to the users.

Some studies focused on allocating the resources, rather than APA [122]-[126]. These methods are appropriate for 
the quasi-static systems and when the LoS blockages are not present. In [122], authors considered both the multihoming and multi-mode mechanisms and they formulated for each mechanism an optimization problem to allocate the resources for maximizing the effective capacity by satisfying the statistical delay target. In the multi-homing mechanism, users can gather the information from the VLC and RF APs at the same time, whereas in a multi-mode mechanism, users can be connected to one type of networks only. Unlike the multihoming mechanism, the centralized formulated optimization problem for multi-mode mechanism needs to select the AP for each user; therefore, a computationally intractable approach was considered in [122], and a distributed suboptimal method was proposed. They showed that, by tightening delay requirements, the multi-homing mechanism provides a much better performance. In [123], for multi-users with multiple VLC APs and one RF AP, authors studied the problem of maximizing the EE under maximum power constraints on both RF and VLC APs, and under QoS constraints, when the multi-homing mechanism was applied. In [124], authors expanded on the work presented in [123] and [125], and jointly allocated the power and bandwidth to the users, but in only one VLC and one RF AP. Both [123] and [124] used Dinkelbach method to convert the nonconvex problem to a sequence of convex problems, then used the sub-gradient method to solve those convex problems. By assuming that a multi-homing mechanism is available to users, there is no need to balance the load by efficiently distributing the users between the RF and VLC APs. In [126], a system consisting of a cascaded power-linecommunication (PLC)/VLC link, along with a RF link was optimized, meaning that the total transmitted power under QoS constraints was minimized. The formulated optimization problem was shown to be a convex problem that could be solved efficiently. In [127], authors formulated a power and sub-channel allocation optimization problem for energyefficient software-defined VLC/RF network, when the users have the multi-homing capability. The optimization problem considered the backhaul constraints, QoS requirements, and the inter-cell interference constraints. With the help of the software-defined controller, the resource allocation strategy can be requested as an application from the application layer, then through the software-defined controller, the requested strategy can be implemented in the APs in the physical layer. Because the objective function is the nonconvex EE function, the Dinckelbach approach was also used to convert the problem into a serial of convex optimization problems.

In [128], a comparison between the performance of the standalone VLC networks with that obtained from augmenting RF APs to the VLC network (in terms of outage probability) was provided. Specifically, authors quantified the minimum required RF resources (bandwidth and power) for the VLC networks to achieve a predefined (per user) rate outage performance. In [129], Tabassum and Hossain used the stochastic geometry to analyze the coverage and the rate of a typical user and compared the results in four types of networks: RF-only, VLC-only, opportunistic RF/VLC (either the user connected to RF or VLC), and the hybrid RF/VLC (the user can gain the resources from both the RF and VLC APs) networks.
Based on several parameters including the FoV receiver, number of interfering LEDs, distribution of the interference, association and coverage probability, and the average rate of the typical users, they found closed-form solutions to distribute the users among the VLC and RF APs. By imposing the QoS constraints based on the data link metrics, i.e. the limits on the buffer overflow and buffering delay probabilities, Hammouda et al. [130] showed that the VLC links offered queuing delays lower than RF links when the data arrival rates at the transmitter buffer were low; however, the RF links supported the higher data arrival rates.

\section{B. Optimizing Multi-User SISO VLC Networks}

As previously shown in III-A, the most common solution for handover, LoS blockages, coverage, and the inter-cell interference is to support the VLC network by a RF network. However, some studies reported in the literature focused on the LB in standalone VLC networks.

In [38], with the help of a central controller, and by considering the arbitrary receiver orientation, Soltani et al. proposed an approach for APA to users, based on the strength of the received signal and the traffic of the APs, aimed at maximizing the system's throughput. Briefly, when a coming user wants to join an established network, the central controller calculates all the offered data rates from all APs and enables the user to select the best AP. In [131], authors jointly allocated time resources to the users and assigned APs to the users. They conceived the problem as a bidirectional allocation game, since the aim of APs is to select the only users that maximize the system throughput, and the users want to select APs providing better QoS. By considering mobile users in standalone VLC networks, Zhang et al. [132] proposed a novel user-to-AP assignment based on anticipating the future users locations and their traffic dynamics, and find a trade-off between the delay and the throughput in the dynamic VLC systems. In [133], authors studied and formulated the joint power allocation and LB problems. By considering a proportional fairness [121], the formulated optimization problem was found an intractable nonconvex. Thus, a suboptimal solution was proposed to optimize both the power and the time fraction in an alternating fashion.

Another factor that can be used to enhance the performance of VLC networks is the arrangement of APs, in which APs are placed and selected in the most appropriate way to improve both the illumination and communication. In [134], authors investigated the effects of the cell size and network deployment on the performance of VLC systems by measuring the signal-to-noise and interference ratio (SINR) distributions, outage probabilities, and data rates. They concluded that the hexagonal cell deployment achieved the best performance, whereas the random cell deployment exhibited the worst performance. In addition, they demonstrated that the multipath effect was much less prominent than the effect of the cochannel interference because of the PD's size compared to the light wavelength. They also compared the performance of the VLC with the RF and mmWave indoor networks and showed the superiority, in general, of the VLC systems. 
The aforementioned papers optimized the VLC networks based on a TDMA scheme. In [135] and [136], the resources in an OFDMA scheme were allocated to maximize the throughput in the downlink LiFi networks. Ling et al. [136] first showed that the problem of allocating the DC bias, the power, and the subcarriers is a coupled problem, and then proposed several algorithms, to compromise between the performance and complexity, starting by proposing an algorithm for allocating the DC bias only, two algorithms for allocating the power and subcarrier jointly, and finally two algorithms to jointly optimize the DC bias, power, and subcarrier. In [135], unlike [136] which considered the subcarriers, authors focused on allocating the time-frequency blocks to increase the flexibility in resource allocation. When allocating the subcarriers, channel responses should be considered, taking into account the fact that the channel quality in the low frequencies is better than that in channels at high frequencies [134]. Because the channel quality depends on the frequency, a careful allocation of the subcarriers (taking the channel into account) leads to a more efficient resource allocation in OFDMA more than that in TDMA.

\section{CoORdinated TRANSMission AND MIMO VLC SYSTEMS}

In this section, we review the references that utilized APs cooperation and MIMO techniques to improve the VLC networks. The APs in VLC networks can work together to beamform the transmitted signals, remove or mitigate interference, improve the space diversity gain, increase coverage, decrease the handover overhead, and decrease the received SNR fluctuations. A coordinated multi-point (CoMP) transmission technique can be implemented by connecting multiple APs through backbone networks so that they can cooperate to design their transmitted signals. Therefore, the joint transmission (JT) can be implemented between the coordinated transmitters to form one cell.

Li et al. [111] studied how the APs should cooperate to mitigate the interference with balancing the load. For managing interference in the $N$ APs and $N$ users system model, the APs in the proposed system in [137], were designed to organize themselves into a cooperative coalition based on the game theory coalition formation. In [138], authors adopted the joint transmission scheme to alleviate the effect of the cochannel interference and to improve the system throughput and the quality of the received signal. In addition to the cochannel interference, the impact of blockages on users can be mitigated using the CoMP joint transmission scheme [139]. Authors of [139] proposed an approach that assigns multiple transmitters to each user, with proportional fairness. Serving a user by multiple LEDs transmitters significantly mitigates the rate of blockages and the handover overhead.

To decrease the backbone traffic and decrease the amount of the exchanged information, authors in [140] coordinated the various transmitters to control interference by either partitioning the resources among transmitters, or by controlling the transmitted power. Partitioning the resources between transmitters decreases the spectral efficiency significantly, even though the inter-cell interference is eliminated [140].
Hence, in [141] and [98], fractional frequency reuse (FFR) was used to trade-off the spectral efficiency for the inter-cell interference, whereas Sun et al. [142] designed the signal for VLC system to trade-off between the interference and the spectral efficiency by imposing time superposition reuse in two neighboring cells, then they proposed an optimal power allocation strategy for this signal design approach. Ma et al. [143] exploited the spatial domain and coordinated the transmission to mitigate interference in a multi-cell MUMISO VLC system, by considering the backbone limited capacity.

Equipping receivers with multiple PDs is an efficient way to improve the received data rate, increase the coverage probability, support mobility, and mitigate the blockages effect. Burton et al. [144] proposed a receiver which is equipped with $L$ PDs, where each one is directed to a different viewing angle and the one with the strongest signal is selected to improve the coverage, decrease the effect of blockage, and support the mobility. Authors in [145] proposed designs for the angle diversity receivers and optimized the angles of PDs orientations for improving the spatial diversity order. Authors in [146] proposed techniques to improve the VLC coverage with the aid of an imaging angle diversity receivers. Using angle diversity receivers, authors of [147] investigated different combining schemes to mitigate the inter-cell interference. In [148], Park et al. designed a mirror diversity receiver that blocks the received light from one direction and strengthens the channel from the other direction, which helps in decreasing the channel matrix correlation.

Because only the non-negative real-valued signals can be transmitted in VLC systems, precoding techniques proposed in literature to CoMP and MIMO VLC networks are different from these investigated in RF networks. Zero-forcing and dirty parity coding were investigated and compared in multiuser multiple-input-single-output (MU-MISO) VLC systems in [149] for maximizing the SINR, whereas zero-forcingbased precoding scheme was proposed in [150]-[152] for minimizing the mean square error. Authors in [153] also used the zero-forcing a precoding approach for maximizing the achievable data rate, whereas authors in [154] proposed a generalized-inverse-based zero-forcing scheme to maximize the max-min fairness and system sum rate.

For the precoding schemes in multi-user-MIMO (MUMIMO) VLC systems, the block diagonalization approach was proposed in [155] to remove interference. Pham et al. [156] used the same precoding approach when considering the non-negativity constraint on the input signal. The TomlinsonHarashima precoding approach was proposed by Chen et al. in [157]; the authors showed it outperforms the block diagonalization approach in terms of BER. A robust linear precoding and receiver design for maximizing the minimum SINR was proposed in [158]. Authors in [159] showed that the dirty paper coding performed better than the linear precoding approaches when the users' CSI are known, whereas the linear precoding approaches are better when only an imperfect users' CSI is available. To mitigate the effects of the indoor VLC channel correlation, authors in [41] calculated a precoding matrix for each subcarrier in a MIMO-MU-OFDM VLC 
TABLE III

PROPOSED TECHNIQUES TO ALLEVIATE THE LIMITATIONS ASSOCIATED WITH VLC NETWORKS

\begin{tabular}{|c|c|c|}
\hline Issue & $\begin{array}{l}\text { Solutions in hy- } \\
\text { brid VLC/RF }\end{array}$ & Solutions in standalone VLC \\
\hline $\begin{array}{l}\text { Small cover- } \\
\text { age }\end{array}$ & $\begin{array}{l}\text { Associate } \\
\text { uncovered } \\
\text { users to RF } \\
\text { network }\end{array}$ & $\begin{array}{l}\text { - Angle diversity receivers } \\
\text { - }[146] \\
\text { - FoV alignment }[162] \\
\text { Efficient AP arrangement } \\
{[134]}\end{array}$ \\
\hline Blockages & $\begin{array}{l}\text { Associate the } \\
\text { blocked user } \\
\text { to RF network } \\
{[114]}\end{array}$ & $\begin{array}{l}\text { - Efficient APA } \\
\text { - Serve each user with } \\
\text { multiple APs [139] } \\
\text { - Angle diversity receivers } \\
\text { [144] }\end{array}$ \\
\hline Handover & $\begin{array}{l}\text { Associate the } \\
\text { unfixed users } \\
\text { to RF network } \\
{[102], \quad[112] \text {, }} \\
{[113],[113]}\end{array}$ & $\begin{array}{l}\text { - Merge VLC APs to be } \\
\text { one cell [111], [163] } \\
\text { - Distribute the APs based } \\
\text { on the anticipated loca- } \\
\text { tion of the user [132] }\end{array}$ \\
\hline Interference & $\begin{array}{l}\text { Associate the } \\
\text { edge-users to } \\
\text { RF network } \\
{[111],[120]}\end{array}$ & $\begin{array}{l}\text { - SDMA [143] } \\
\text { - Frequency reuse [111] } \\
\text { - Fractional frequency } \\
\text { reuse [98], [141] } \\
\text { - Fractional time reuse } \\
\text { [142] } \\
\text { - Joint transmission [111], } \\
\text { [138] } \\
\text { - APs arrangement [137] } \\
\text { - User-centric network de- } \\
\text { sign [91], [163] } \\
\text { - Efficient resource and } \\
\text { power allocation [140] } \\
\text { - Angle diversity transmit- } \\
\text { ters [147], [164] } \\
\text { - Angle diversity receivers } \\
\text { [165] }\end{array}$ \\
\hline $\begin{array}{l}\text { Limited } \\
\text { LEDs } \\
\text { bandwidth }\end{array}$ & $\begin{array}{l}\text { Equipping the } \\
\text { users with } \\
\text { multi-homing } \\
\text { capability } \\
\text { to gather } \\
\text { information } \\
\text { from RF } \\
\text { and VLC } \\
\text { simultaneously } \\
{[124]}\end{array}$ & $\begin{array}{l}\text { - Efficient LED design } \\
\text { [33] } \\
\text { - Extensive frequency } \\
\text { reuse [120] } \\
\text { - Densify the APs [166] } \\
\text { - Employ NOMA [167] } \\
\text { - Angle diversity transmit- } \\
\text { ters [164], [165] }\end{array}$ \\
\hline
\end{tabular}

system by exploiting the phase differences, after transforming them to a frequency domain of different links. The precoding matrix was designed to eliminate the inter-user interference. Cai et al. [160] proposed algorithms of PD selection in imaging receivers to mitigate the channel correlation and decrease the BER in a MU-MIMO-OFDM VLC system. By exploiting the knowledge of the transmitted symbols, authors of [161] proposed an adaptive precoding scheme to only eliminate the destructive interference and correlate the constructive interference. Designing the precoding matrix to correlate the constructive interference provides a significant improvement, in terms of BER, compared to the zero-forcing precoding approach [161].

Space division multiple access (SDMA) has been proposed in VLC networks to mitigate effects of interference and to improve the spectral efficiency [164]-[166], [168], [169]. In the SDMA scheme, multiple LEDs are designed to generate spatially separated beams that are directed to various users. Kin and Lee [168] showed experimentally that the SDMA efficiently can improve the amplitude of the received signal. In [169], authors proposed a low complexity algorithm (compared to the exhaustive search algorithm) named 'random pairing algorithm' for grouping the users into multiple SDMA groups in order to obtain a better area spectral efficiency under users' fairness constraints. Each user group is served by multiple coordinating APs when applying the zero-forcing precoding method to eliminate the inter-cell interference. In [164] and [165], authors proposed the use of angle diversity transmitters to increase the bandwidth and mitigate the interference. The authors estimated the performance of a SDMA-VLC system by deriving the analytical upper and lower bounds of the average spectral efficiency. When the number of LEDs is much larger than the number of users, where multiple LEDs can serve one user, and using the angle diversity transmitters proposed in [165], authors in [166] addressed the problem of properly assigning multiple LEDs for each user. A power allocation algorithm was also proposed to improve the sum rate and the system's fairness.

The user-centric (UC) design is the most appropriate approach for forming the cells when the number of APs is higher than the number of users. In [170], Zhang et al. investigated the user centric design for VLC, for which the cells' structures are not with regular shape. First, the users were clustered to multiple clusters, then the APs were distributed to the clustered users. In [171], Li et al. used the UC design to improve the system fairness by proposing algorithms aimed at scheduling users and maximizing the sum utility of the system. In [91], in addition to forming the cells and associating the APs, the powers were allocated to the clustered users to maximize the EE. In [172], authors used these techniques of cell formation and power allocation to design energy-efficient scalable video streaming with considering an adaptive modulation mode assignment. The common clustering approach used in [91], [171], [172] is the edge distance clustering. After the users are clustered, the APs are assigned to the clustered users, using an anchoring AP association approach. Finally, the power at the APs is allocated to the associated users to maximize the EE.

Authors of [163] and [173] showed that the procedures user clustering, AP association, and power allocation are joint problems, when the EE maximization is the target. Hence, they proposed a novel user clustering method to maximize the separation between clusters and help in reduce the intercell interference. They then proposed a joint power allocation and AP association to maximize the EE.

Table IV summarizes the optimization techniques, which were proposed in literature to minimize the effect of VLC disadvantages and reviewed in Sections III and IV. In Table IV, we outline the solutions for the VLC disadvantages in hybrid RF/VLC networks and standalone VLC networks. 


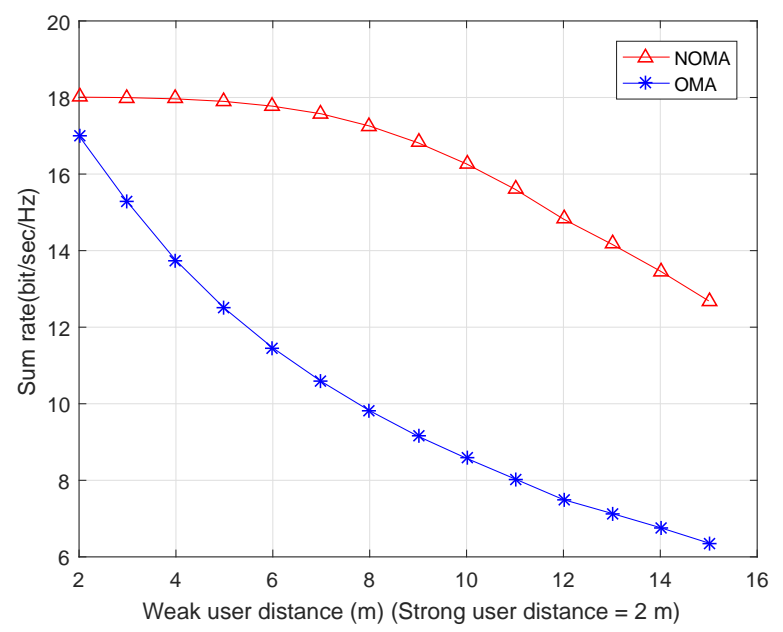

Fig. 8. The impact of increasing the distance of the weak user with achieving the fairness constraint that both users receive equal data rate, when $\mathrm{FoV}=$ 40 , incidence angle $=0$, and irradiance angle $=0$.

\section{NOMA IN VLC}

In this section, we introduce NOMA, a new technology nominated for the fifth generation $(5 \mathrm{G})$ wireless networks aimed at increase the throughput, decrease the latency, and improve the fairness and connectivity. The rational behind NOMA is the use of a single resource component by multiple users, whether this component is a sub-carrier, a time slot, or a spreading code. With this basic concept, different types of NOMAs, such as the power domain NOMA (PD-NOMA), pattern division multiple access (PDMA), sparse code multiple access (SCMA), were presented as good candidates for the 5G multiple access technique. More details on NOMA in traditional RF networks are provided in [174], [175].

In VLC networks, researchers are interested only in power domain NOMA (PD-NOMA). The goal of PD-NOMA is to set different power levels for different users. For instance, for two users served by the same base station (BS), and using the same OFDM subcarriers, the BS assigns a high power to the user with poor channel and a low power for the user with a better channel. In other words, assuming that $h_{1}>h_{2}$, where $h_{i}$ is the channel of the $i^{t h}$ user, the BS transmits the signal of User 2 with higher power. User 2 decodes the received signal and treats User 1's signal as noise, whereas User 1 first decodes the signal of User 2, and then removes it from the received signal, after that it decodes its own signal. To generalize this idea, we assume that we have $N$ users served by the same BS, and first categorize them based on their channel gains as $h_{1} \leq h_{2} \leq \ldots \leq h_{N}$. When using the NOMA technique, the BS transmits the signal of all users using same carrier, and the received signal, at the $k^{t h}$ user, can be expressed as follows:

$$
y_{k}=h_{k} \sum_{j=1}^{N} \alpha_{j} \sqrt{P} s_{j}+n_{k},
$$

where $\alpha_{j}$ is the power coefficient of the user $j, s_{j}$ is the information signal of the user $j$, and $n_{k}$ is the additive white

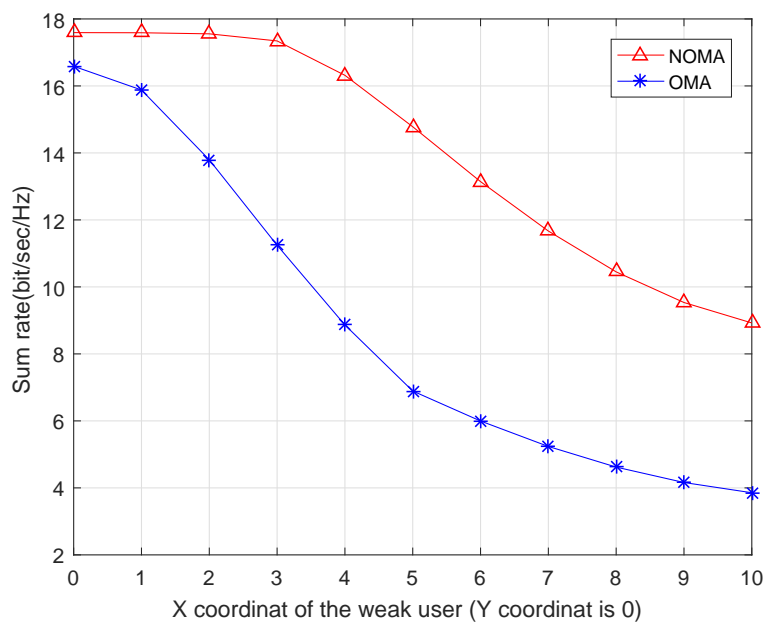

Fig. 9. Shifting the weak user in the $\mathrm{X}$ coordinate where the incidence and irradiance angles changes accordingly, the strong user located at $(0,0)$ coordinate, $\mathrm{FoV}=40$.

Gaussian noise. According to NOMA, users with a lower channel gain will have a higher power, meaning that $\alpha_{1} \geq$ $\alpha_{2} \geq \ldots . \geq \alpha_{N}$. Then, the successive interference cancellation is implemented to decode the signals received by the users. In other words, User $N$ must decode all the signals of all users to have its own signal, and User $N-i$ has to decode $N-i$ signals to obtain its intended signal. It is clear that, as the number of users increases, the complexity of decoding the signal is increased. In addition, the residual interference coming from inaccurate channel estimation increases with the number of users.

Implementing the NOMA in VLC networks requires considering the unique properties of VLC networks such as the limited bandwidth of LEDs, the maximum transmit power that is restricted by the illumination requirements, the blockages that make the channel between the transmitter and receiver close to zero, and the dramatic deterioration in the channel, as the distance increases. In addition, the channel value can be controlled by changing the FoV of the receivers or the semi-angles of the transmitters (if they are tunable), and these two factors can be selected to improve the performance of NOMA-VLC networks. Because the PD-NOMA scheme is based on successive interference cancellation (SIC), NOMAVLC networks require all users' CSIs to be available, which is the case in VLC. It was also shown that the NOMA scheme performance is enhanced as the SNR increases [181], which is the case of VLC link. These features offered by NOMA-VLC networks led many researchers to investigate these networks and find out how the NOMA outperforms OMA schemes in VLC systems.

In Figs. 8 and 9, we consider a system model consisting of one VLC AP fixed in the ceiling and two users in the floor. We assume that one user is located directly under the VLC AP with $2 \mathrm{~m}$ distance which is considered the strong user. Figs. 8 and 9 present simulation of how the NOMA outperforms OMA in VLC networks, when only the distance of the weak user increases (Fig. 8) and when the distance, 
TABLE IV

SUMMERY OF NOMA-VLC PROPOSED SYSTEMS

\begin{tabular}{|c|l|l|l|}
\hline Ref. & Sysem Model & Main Objective & Techniques and Characteristics \\
\hline \hline$[176]$ & Two APs \& three users & Achievable throughput & $\begin{array}{l}\text { GRPA to allocate the power, tuning users FoV and } \\
\text { LEDs angels transmission }\end{array}$ \\
\hline$[177]$ & One AP \& $K$ users & $\begin{array}{l}\text { Evaluating } \\
\text { in terms of NOMA } \\
\text { probability and ergodic } \\
\text { capacity }\end{array}$ & $\begin{array}{l}\text { Evaluating NOMA and comparing it with OMA, } \\
\text { careful selecting LED with adjusting its transmission } \\
\text { angel improves the performance }\end{array}$ \\
\hline$[178]$ & One AP \& $K$ users & $\begin{array}{l}\text { Achievable sum-rate with } \\
\text { fairness }\end{array}$ & $\begin{array}{l}\text { Convixifing the non-convex optimization problem } \\
\text { and solving it using the dual decomposition method }\end{array}$ \\
\hline$[179]$ & One AP \& $K$ users & Achievable sum-rate & $\begin{array}{l}\text { User grouping, interference mitigation, and power } \\
\text { allocation }\end{array}$ \\
\hline$[139]$ & $\begin{array}{l}2 \times 2 \text { MIMO, one AP \& } \\
K \text { users }\end{array}$ & Achievable sum-rate \\
\hline$[180]$ & One AP \& $K$ users & $\begin{array}{l}\text { Reducing computational } \\
\text { burden and overhead }\end{array}$ & $\begin{array}{l}\text { Limited feedback schemes, when users change their } \\
\text { locations and vertical orientation }\end{array}$ \\
\hline
\end{tabular}

incidence, and irradiance angles change (Fig. 9). 'Strong' and 'weak' users mean the user with the best channel and the user with the worst channel, respectively. In [182] and [183], authors showed the superiority of NOMA over OFDMA, in VLC systems, with respect to sum rate and BER performance, respectively. In order to allocate the power that maximizes the sum rate, authors of [184] optimized the NOMA-VLC downlink for a two-user and one AP system, with satisfying certain QoS constraints.

In [176]-[178], [180], [185], authors evaluated the performance of the NOMA-VLC for one VLC AP and multiple users. In [177], [185], authors presented distribution functions for the uniformly distributed users, then evaluated the NOMAVLC system by comparing it to OMA-VLC system in two case scenarios: 1) when each user has a data rate target, and 2) when the data rates of all users are assigned opportunistically according to their channels. By considering the proportional fairness [121], authors of [178] showed that the formulated problem was of non-convex type, but could be converted to a convex problem that could be solved using a dual decomposition method. Authors of [180] evaluated and compared the NOMA and OMA schemes, when the users change their locations and their vertical orientation. Instead of reporting the full CSI that increases the computational complexity, they used limited-feedback schemes to categorize users based on their mean vertical angle and mean distance, and this might be most appropriate to simplify the implementation.

For multiple APs, authors of [176] studied NOMA-VLC networks when the network consisting of two VLC APs and three users. They proposed a gain ration power allocation (GRPA) approach to allocate power to the various users, and compare it with the static power allocation approach; assuming that the users' movement is assumed to follow random walk model. In the GRPA approach, the power for the user $k$ is assigned to be $P_{k}=\left(\frac{h_{1}}{h_{k}}\right)^{k} P_{k-1}$. Assuming the users' FoV and the transmission angels of LEDs are tunable provides a potential to improve the performance significantly. For multicell VLC networks, under the assumption that the frequency reuse FR $=2$, the users in [179] were grouped based on the received interference. If any user suffered from interference, they were given a special resource blocks, and NOMA was implemented for the remaining users. For the users sharing the same resource block, the authors formulated optimization problems to allocate the power, under QoS constraints, and provided solutions to the formulated problems.

NOMA has been also used in MIMO VLC systems [186], [187]. In [186], authors experimentally investigated a system with single carrier mode of transmission, using the frequency domain SIC, but without considering the power allocation problem. In [187], Chen et al. proposed a power allocation algorithm (called normalized gain difference power allocation (NGDP) approach) aimed at reducing the complexity and increasing the efficiency of 2x2 MIMO-NOMA-VLC systems with multiple users. In their study, they classified the users using the sum of the channel gains for each user, with respect to each LED. In the proposed power allocation method, the power allocated to the user $k$ and user $k+1$ in the $i$ th LED are related: $P_{i, k}=\left(\frac{h_{1 i, 1}+h_{2 i, 1}-h_{1 i, k=1}-h_{2 i, k+1}}{h_{1 i, 1}+h_{2 i, 1}}\right)^{k} P_{2 i, k+1}$, where $h_{j i, k}$ is the channel between the $i$ th LED and the $j$ th PD of the $k$ th user.

For the uplink VLC systems, authors of [188], [189] introduced a phase pre-distortion method to decrease the uplink error rate performance in NOMA-VLC systems. Since in [188], authors used the SIC to decode the signals, whereas in [189], a joint detection method was used to improve the system performance. Table IV summarizes the works conducted in NOMA-VLC systems by presenting the studied system models, the main objective, and the techniques used to achieve the established objectives.

\section{ENERGY HARVESTING IN VLC SYSTEMS}

Much attention has recently been paid to energy-harvesting techniques at user-equipment devices, either from exploiting the surrounding environment, or by transferring wireless power. Energy harvesting is the capability of converting the radio frequency (RF) signals or light intensity into electrical voltage/current. With the advent of the era of the IoT, the demand for transferring the power and enabling IoT devices to harvest energy using light or RF transmission is increasing, especially in indoor applications where smart buildings, health monitoring, and sensors devices applications become abundant. Extensive work has been done to design, evaluate, and optimize simultaneous wireless information and power transfer in RF networks [190], [191]. Work on harvesting 


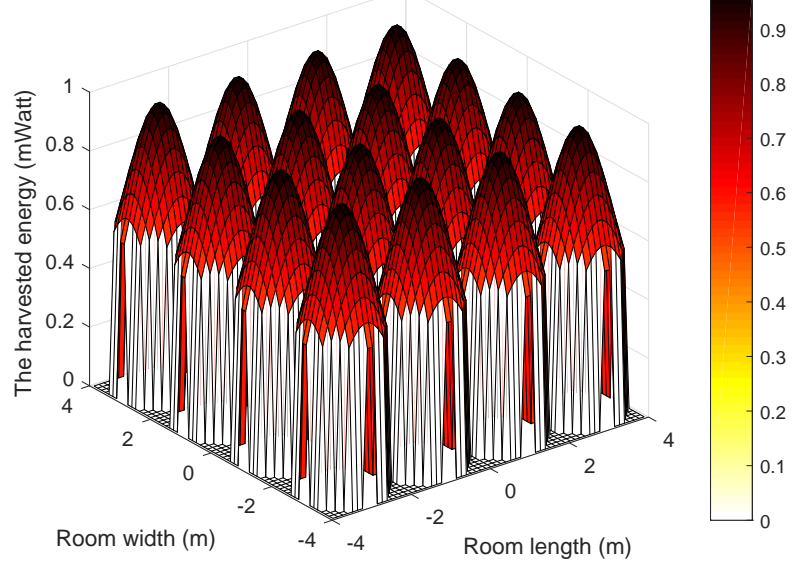

(a) $\mathrm{FoV}=30^{\circ}$

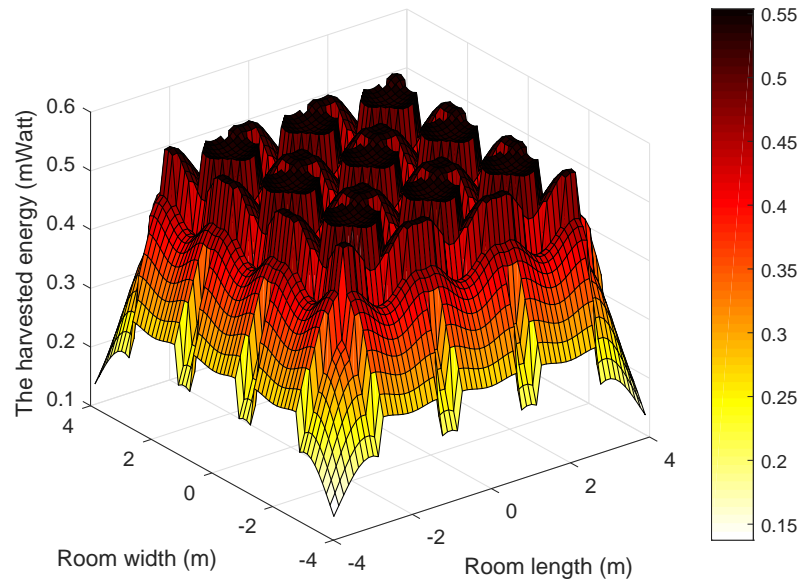

(c) $\mathrm{FoV}=50^{\circ}$

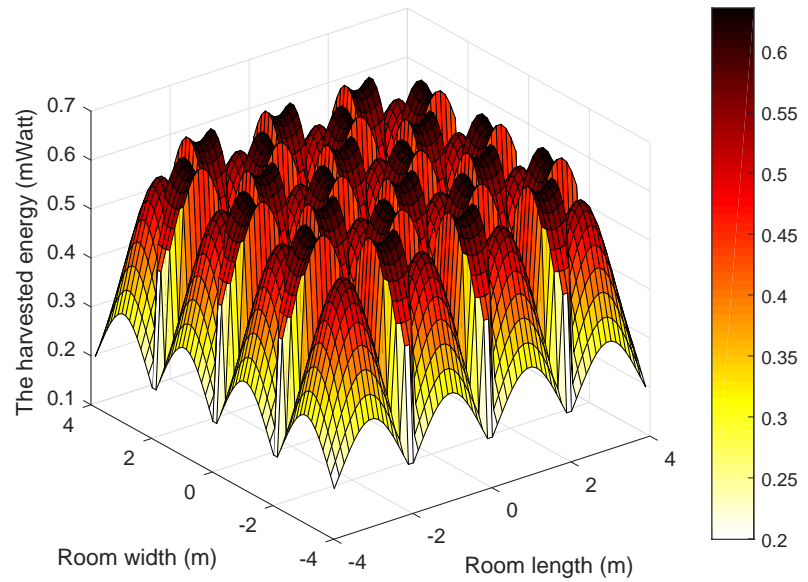

(b) $\mathrm{FoV}=40^{\circ}$

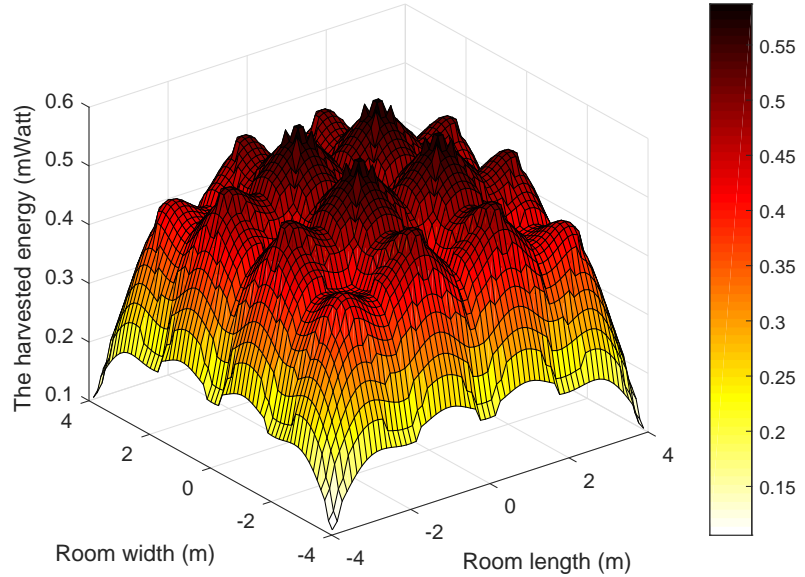

(d) $\mathrm{FoV}=60^{\circ}$

Fig. 10. The distribution of the possible harvested energy over the area, with diferent user's FoV, where the system model consists of 16 VLC APs.

the energy and transmitting the information using the light is scarce, as it is still in its early stage.

Different from harvesting the energy in the RF networks, the energy can be harvested using the DC component that is transmitted along with the information signal to ensure the non-negativity of signals. This DC component can be easily separated from the modulated signal using capacitor and goes to the energy harvesting circuit. Since the recent solar cell panels can provide more than $40 \%$ conversion efficiency [192], a new trend has emerged in the research community for using solar cells at the receivers to detect information signal and harvest energy.

Investigating the harvesting of energy in VLC systems has been a timely topic of interest [99], [193]-[199]. A few papers recently published proposed to investigate systems that use the light to jointly transfer power, meet illumination requirements, and transmit data. Authors of [99] experimentally harvested the solar energy with mobile phone by equipping it with a commercial solar panel in an indoor environment. They showed that the devices directly exposed to the indoor light could be charged to a satisfactory level. In Fig. 10, we conduct a simulation to show the amount of the possible energy, which can be harvested by a user, over the surface of an $8 \times 8$ room with 16 VLC APs. In these figures, we consider the same system model that was considered in Fig. 3 with energy harvesting users that are equipped with solar cells. Equations (1) and (18) with the given simulation parameters in Table II are used to find the received harvested energy at each point in the floor area. As observed in this figure, the user's FoV has an impact on the harvested energy because it affects the channel quality and the system coverage.

Authors of [193] investigated the concept of indoor optical wireless power transfer to solar cells during darkness hours. By using laser diodes and a solar panel, they measured the power efficiency and showed an improvement over the inductive power transfer systems, of approximately 2.7 times. By using 42 laser diodes, they claimed to deliver $7.2 \mathrm{~W}$ of optical power to a solar panel $30 \mathrm{~m}$ distant from the diodes. Authors of [203] studied how much artificial indoor light could deliver an amount of energy, using different types of 
TABLE V

SUMMERY OF THE ENERGY-HARVESTING SYSTEM MODELS AND TECHNIQUES

\begin{tabular}{|c|l|l|l|l|}
\hline Ref. & System Model & $\begin{array}{l}\text { The } \\
\text { harvested } \\
\text { power }\end{array}$ & Main Objective & Techniques and Characteristics \\
\hline \hline$[194]$, & $\begin{array}{l}\text { One transmiter, one } \\
\text { relay, and one re- } \\
\text { ceiver }\end{array}$ & DC-current & $\begin{array}{l}\text { Data rate and packet } \\
\text { loss probability }\end{array}$ & $\begin{array}{l}\text { The relay harvests the energy using the visible light } \\
\text { then forwards the signal through RF link to the } \\
\text { destination }\end{array}$ \\
\hline$[196]$ & $\begin{array}{l}\text { One AP \& } K \text { users } \\
\text { time slot and harvests the energy within the time } \\
\text { slots assigned to other users }\end{array}$ & AC-current & Sum-rate & $\begin{array}{l}\text { Allocating the optical intensity and the time slots } \\
\text { subject to a given harvested energy constraint }\end{array}$ \\
\hline$[197]$ & $\begin{array}{l}\text { One AP \& } K \text { users in } \\
\text { outdoor }\end{array}$ & AC-current & Sum-rate & $\begin{array}{l}\text { A time slot is devoted to transfer the power for the } \\
\text { users to be used then for the uplink transmission }\end{array}$ \\
\hline$[198]$ & $\begin{array}{l}\text { One AP \& } K \text { users } \\
\text { over the uplink }\end{array}$ & AC-current \\
tion
\end{tabular}

receiving cells.

In [194] and [195], a dual-hop hybrid VLC/RF communication system was studied as a means to reach out to the out-ofthe-coverage user. The authors showed that visible light could be used, in the first hop, to transfer both data information and energy to the relay. The relay, could then forward the data to the destination, using the harvested energy. In [196], [197], authors maximized the sum-rate utility of a VLC system consisting of one AP and $K$ users, subject to individual QoS constraints. Li et al. [196] assumed that a user $k$ can receive the information in their assigned time slot, and the power within the time slots assigned to other users. In [197], on the other hand, Abdelhady et al. proposed solving the problem by allocating the optical intensity and time slots, using an upper bound on the individual required harvested energy. Authors in [198] characterized the outage performance of a hybrid VLCRF system, where the visible light is used for the downlink to transfer the energy and data to the users, who then use the harvested energy to transmit a RF signal in the uplink.

All the studies mentioned above use the alternating current (AC) component for harvesting the energy, where the DC component of the transmitted light is fixed and readily used to harvest energy [34]. In [34], authors designed an optical wireless receiver using a solar panel and enabled it to receive information and harvest energy simultaneously. Because the received current signal contains both DC-current and the ACcurrent components, authors in [34] suggested to attenuate the $\mathrm{AC}$ current, using an inductor to remove the ripples from the DC-current that is forwarded to energy harvesting branch, and to block the DC-current, using a capacitor, to obtain only an AC-current in the communication circuit. Sandalidis et al. [200] investigated the three functions of the LED lamp, i.e. the illumination, communication, and the energy harvesting, on a system consisting of a desk LED close to the receiver equipped with a solar cell. The authors divided the received optical power between the information signal and the harvested energy, using a splitter.

Diamantoulakis et al. [199] studied the lightwave information and power transfer for a system consisting of one transmitter and one receiver. They provided the two following protocols to maximize the harvested energy at the receiver, under data rate constraint: 1) Splitting the time into two portions, one dedicated to maximize the user's SINR, and the second assigned purely to maximize the harvested energy by maximizing the DC component, 2) Optimization of the DC bias, in phase 1, to maximize the harvested energy under QoS constraints, with phase 2 assigned only to harvesting the energy. However, optimizing the DC bias for the whole time is more general, and there is no need to split the time between harvesting the energy and transmitting the data. In addition, the formulated problem would be more challenging if there were multiple receivers, since the fairness, in terms of data rate and harvested energy, is required. Hence, authors in [201] studied the simultaneous information and power transfer in MISO multi-user VLC system, where some users are interested only in gathering information and the others users interested in harvesting energy. The problem of maximizing the total harvested energy under QoS constraints was formulated as a non-convex problem, and hence, a special iterative algorithm was used to solve the formulated problem.

Table V summarizes the studied system models and techniques used for harvesting the energy in VLC systems.

\section{SECURING VLC SYSTEMS}

Traditionally, the mission of the physical layer researchers and designers is to provide a reliable signal transmission to the relevant receivers, while the mission of securing and protecting the transmitted information is usually assigned to the upper layers of the networks [204]. However, with the spreading of the broadcast-nature networks and with the seeking for higher data rates, researchers have found with new 
techniques to build secure communication networks, using the physical layer.

The physical layer security (PLS) deals with how to exploit the randomness of noise, channels, and different resources, such as multi-antenna and cooperative nodes, to minimize the information that can be extracted by the eavesdropper [205], [206]. The secrecy capacity was introduced by Wyner [207] as a metric to measure the security performance; it has been defined as the largest data rate that can be acquired at the authorized receiver, with having the eavesdropper completely unaware of the transmitted information. To have a precise quantification of the security, the eavesdroppers are considered knowing the network parameters and have a sufficient computational capability.

In this section, we review the work that has been performed on the PLS, in VLC networks. For the PLS in RF systems, readers can refer to [208] and [209].

As mentioned in the Introduction Section VLC networks are more secure than RF networks because the light is blocked by any opaque objects. Nevertheless, securing VLC networks is required, specifically in public areas where the the emitted light can be receive by multiple receivers. In these public areas, possible eavesdroppers may be existed and trying to attain confidential information [210].

Many existing studies addressed the PLS in VLC systems, and different mechanisms were proposed to investigate and improve the secure communications. The proposed techniques generally depend on different network parameters, such as the availability of the eavesdroppers' CSI, the number of LEDs equipped with transmitters, the number of the legitimate users and eavesdroppers. These techniques use a zeroforcing precoding approach that eliminates the transmitted information to eavesdroppers; they may use artificial noise or jamming aimed at confusing eavesdroppers, they build a protected zones using angle diversity transmitters, optimizing the input distribution, characterizing the security in VLC systems using the stochastic geometry. The artificial noise and signal modification are possible in real-world VLC applications, but the MAC layer cannot provide sufficient protection against eavesdropping [211].

In [212], Ayman Mostafa and Lutz Lampe started investigating the PLS in MISO-VLC channel with one eavesdropper and one authorized user. When the CSI of the eavesdropper is available, the zero-forcing precoding approach is applied, but if the CSI is unknown, the transmitter divides its own optical power into two portions, a one used for the informationbearing signal and the other used for emitting jamming signals to confuse eavesdroppers without affecting legitimate receiver. It is important to note that the zero-forcing preceding approach can be implemented when the number of LEDs transmitters is larger than the number of eavesdroppers. Similarly, the jamming signals can be eliminated, at the legitimate users, when the number of users is lower than the number of LEDs jammers.

Instead of dividing the power between information and jamming signals, Ayman Mostafa and Lutz Lampe [76] assigned one LED for transmitting data and assigned all the others to transmit jamming signals. To eliminate the jamming signal at the legitimate receiver, different LED jammers coordinate their transmitted signals to be eliminated at the legitimate receiver. In [213], authors devoted some LEDs to transmit the information and keeping the others for emitting an intrusion signal and shape a protected zone where potential eavesdroppers receive degraded signals.

In [81], authors solved two optimization problems to find the optimal beamforming vector at LEDs, when CSI of the eavesdropper is known and when the transmitter has a limited information about the location of the eavesdropper. For amplitude-constrained wiretap channels, the secrecy rate maximization problem was formulated as a non-convex optimization problem when the CSI of the eavesdropper is known [214]. When the CSI of the legitimate user is limited, and the CSI of the eavesdropper is not available, the authors estimated the eavesdropper channel by deriving the uncertainty sets that reflect the inaccurate knowledge of the eavesdropper location, orientation, LEDs half angle, and non-LoS components. In [215], a joint beamforming and jamming approach was proposed and optimized to improve the PLS for one legitimate user with multiple eavesdroppers in the MISO-VLC system. Two vectors were optimized jointly: the beamforming vector that is multiplied by the legitimate information, and the jamming precoding vector that is multiplied by the jamming signal, in order to maximize the SNR of the legitimate user, under SNR constraints for both perfect and imperfect CSIs at the transmitter.

In [216] and [217], V. Pham and T. Pham investigated ways of transmitting confidential messages to two different legitimate users, in a MISO-VLC system, while keeping the messages confidential from each other (as well as the eavesdropper). The zero-forcing precoding approach was applied to guarantee the confidentiality among users. Authors in [218] proposed a new precoding scheme to send $N$ confidential messages to $N$ destinations. This precoding scheme is based on finding the precoding matrix from the eigenvectors associated with largest eigenvalues of the different $N$ VLCMISO channels. In [78], authors studied the PLS in a system consisting of massive low-intensity LEDs, distributed uniformly in the ceiling, multiple legitimate receivers, and multiple eavesdroppers with limited CSI. After defining the insecurity zone around the legitimate receivers, they designed the beamformer so that it can direct its main lobes towards the defined zones and minimize the information rate outside the defined insecurity zone.

When the VLC system contains multiple eavesdroppers distributed randomly in the considered area, the stochastic geometry can be used to characterize the system and to provide analytical expressions of the secrecy outage probability and of the average secrecy rate. In [219], authors studied the PLS in one VLC cell consisting of group of LED lamps located in the center of the ceiling, with one receiver and multiple randomly distributed (with the Poisson point process (PPP)) eavesdroppers. They provided closed-form analytical expressions for the secrecy outage probability and the average secrecy rate, using the stochastic geometry method under the assumption that the floor area is a circle.

In [220], Cho et al. proposed a LED selection scheme 
to improve the secrecy outage probability, when the eavesdroppers are randomly located in the selected area, and their CSI are unknown to the transmitters. Similarly to what was done in [219], they used the PPP to model the randomness of the location of the eavesdroppers. The same authors used the proposed eavesdropper location modeling to analyze the performance of the MISO-VLC system model and proposed a beamforming solution [221]. To find the optimal beamforming vector, they formulated the following optimization problems: minimizing the average eavesdropper SNR under a given SNR constraint to the legitimate receiver, maximizing the authorized user SNR under the average eavesdropper SNR constraint, and they optimized the same problems for the rate, instead of the SNR. In all the formulated problems, closedform expression were provided for the beamforming vector solution. Because the VLC LEDs provide a very limited coverage area, the beamforming solution for the secrecy maximization can be approximated to be a LED selection. By selecting the AP closest to the legitimate user, secrecy rate would then be improved, as the distance between the two users increased [221]. The same authors studied the PLS in a VLC system when different eavesdroppers combine the received observations using maximal ratio combining (MRC) approach, to degrade the secrecy performance [222]. The authors used the stochastic geometry to anticipate the secure communication under a pre-defined eavesdroppers' density.

In [223], Yin and Haas considered the unique properties of the VLC channel and the VLC network's layout to fully characterize the secrecy outage probability and the ergodic secrecy rate in multiuser (multiple legitimate users and multiple eavesdroppers), multi-cell VLC systems. The APs in the ceiling were modeled using a two-dimensional homogeneous PPP, under the assumption that some of the LEDs are not working as VLC APs (they provide only the illumination), whereas both users and the eavesdroppers are modeled using another independent two-dimensional homogenous PPP at their plane. They investigated the secrecy performance for three scenarios: 1) the legitimate user is served by the nearest AP, 2) the legitimate user is served by cooperating APs, and 3 ) the legitimate user is located in the protected zone around the AP, where the eavesdropper is not allowed to be.

Because all the aforementioned work ignored the fact that an eavesdropper might exploit the reflected light to obtain unauthorized information, authors of [224] considered the effect of the reflected path and the channel correlation to propose an eavesdropping-resilient framework for VLC security. Authors of [225] examined the contribution of the small gaps beneath the doors or kay holes in helping eavesdroppers. They also concluded that eavesdroppers could gain information from reflected lights from the walls. Cho et al. [226] showed how the non-LOS or the reflected light would affect the secrecy outage probability in a system model consisting of multiple LED transmitters, one legitimate user, and multiple randomly distributed eavesdroppers. They showed that the secrecy outage probability depends on the position of the legitimate user, the design of the LED transmitters, and the location of the eavesdroppers with respect to the reflecting points.
For MIMO-VLC systems, in [227], a MIMO system was used to establish a secure communication zone by minimizing the BER in the protected zone and maximize it everywhere else. In [228], authors studied the PLS in a MIMO-VLC system model consisting of one transmitter equipped with multiple LEDs, one multiple-PD eavesdropper, and one multiplePD authorized user. In both cases (the channel information of the eavesdropper is available or not), they derived the optimal covariance matrix and the optimal signaling scheme for secrecy capacity maximization. Authors of [229] improved the PLS in VLC systems by applying angle diversity transmitters that are effectively minimizes the leak of information. By comparing different types of optical network deployments, they concluded that the hexagonal deployment is the best in terms of secure communications, whereas the PPP deployment is the worst. Wang et al. [230] proposed LED pattern selection scheme to improve the secrecy performance of the generalized space-shift keying VLC systems.

For the hybrid VLC/RF networks, authors of [231] studied the PLS when the network consisted of one RF AP, one VLC AP, one legitimate receiver, and one eavesdropper. Both the eavesdropper and the receiver have the multi-homing capability. The RF and the VLC AP are equipped with multiple antennas and multiple LEDs, respectively. By optimizing the beamforming vectors and the transmit powers at both the VLC and RF APs, they formulated their problem as minimization of the total consumed power under having the aggregated information rate at the eavesdropper nulled and having the information rate at the receiver above a predefined threshold. In [232] and [233], authors derived the secrecy outage probability for the uplink in a system consisting of one legitimate receiver, one eavesdropper and two RF VLC APs. The downlink was implemented by the VLC AP, where the energy is harvested by both receivers using the visible light. Then the legitimate user use the harvested energy to transmit data through the RF link, while the eavesdropper is trying to acquire the transmitted information. They concluded that increasing the circle area or decreasing the LED height improves the secrecy outage probability.

In [234], Mukherjee investigated the lower and upper bounds of the secret-key capacities in SISO VLC system, and analyzed the secret-key transmission scenario in MISO VLC system. Al-Moliki et al. proposed a security protocol that generates confidential keys dynamically from the bipolar real OFDM samples to encode each signal frame by using the cyclic prefix samples placed in the small channel impact area [235], [236]. The same authors upgraded their proposed protocol in [235] to combat the known-plain text attacks and chosen-plaintext attacks by applying logistic chaotic maps to the system [237].

A chaotic channel determined subcarrier shifting (CDSS) scheme with pre-equalization were presented in [238] to improve the PLS in DCO-OFDM VLC system. For secure image transmitting in VLC channel, chaos scrambling schemes were proposed in [239] for discrete-Foruire transform precoded OFDM-based, and in [240] for discrete cosine transform precoded OFDM-based systems. 
TABLE VI

TECHNIQUES USED FOR PLS

\begin{tabular}{|l|c|}
\hline State & Appropriate techniques \\
\hline Full CSI is available & $\begin{array}{l}\text { Z Zero-Forcing precoding [212] } \\
\text { - Design the beamforming matrix from } \\
\text { the channel matrix eigenvectors [218] } \\
\text { - LED selection [221] }\end{array}$ \\
\hline $\begin{array}{l}\text { Location of the eaves- } \\
\text { dropper is available }\end{array}$ & $\begin{array}{l}\text { - Build a protected zone where eaves- } \\
\text { dropper cannot be located [78], [223] }\end{array}$ \\
- Jam a defined zone where the eaves- \\
dropper can be located [213] \\
- Estimate the eavesdroppers' channel \\
and use the same techniques when \\
CSI is available [81], [214] \\
- Use the angle diversity transmitters \\
[229], LED selection [230], and APs \\
arrangement [229]
\end{tabular}

\section{Summary ANd Open Research Problems}

In this paper, we reviewed the optimization techniques that proposed to improve the performance of VLC networks and minimize the effects of the VLC limitations. The review covered the different types of VLC networks: hybrid VLC/RF networks, VLC standalone networks with APs' coordination, NOMA-VLC networks, VLC networks that contain energy harvesting users, and VLC networks that contain eavesdroppers.

Based on the existing work in the literature, in this section, we outline different challenges and open research problems that need to be considered and investigated in the future work.

\section{A. Capacity Derivation, Load Balancing, and CoMP Trans- mission}

- For the optical intensity channel capacity, we recommend to consider some practical constraints in deriving or investigating the optical channel capacity such as the synchronization error, random device orientation, imperfect channel estimation, delayed feedback, and shadowing.

- Users' FoV alignment for balancing the load: we show in Section III that many studies have been dedicated to balance the load in VLC or in hybrid VLC/RF networks. These studies focused mainly on optimizing the users' association, time or bandwidth resource allocation, and the power allocation. However, as we show in Section II-D6, the users' FoV parameter has a great impact on the system coverage, inter-cell interference, handover overhead, and on channel quality. Therefore, optimizing the users' FoV jointly with distributing the users and allocating the power would be more effective in balancing the load and in maximizing the system utilities.
- Cooperation among users in VLC system: as mentioned earlier, the VLC systems suffer from SNR fluctuation, where some users would receive a high QoS and others would receive a poor QoS because of the limitedcoverage feature, LoS blockages, inter-cell interference, and handover overhead. Here, we propose one solution which is based on establishing a cooperation among users. Specifically, all the users can be served by the VLC APs and the good-serviced users can cooperate to help the poor-serviced users through RF links. That means the good-serviced users should also have the messages that must be forwarded to the weak-serviced users and this can be implemented by following the principle of cooperative-NOMA.

- User-centric design in hybrid VLC/RF systems: different papers optimized the user-centric cell design in standalone VLC networks [91], [163]. However, optimizing the user-centric cell design in hybrid RF/VLC networks would be more challenging and leads to a high impact in improving the system utilities. The problem can be divided into two interlinked problems, which are assigning the users to networks (either RF or VLC) and clustering the users and assigning the APs in each network. These two problems can be solved alternatively to improve the system utility and to achieve the required constraints.

- Optimizing the joint cell formation and power allocation in user-centric VLC design: it was shown that the procedures user clustering, APs association, and power allocation are interlinked problems [163], but only a subboptimal approach has been proposed for jointly associating the APs and allocating the power. However, formulating an optimization problem that considers the three procedures and proposing solutions for the formulated problem would be more efficient in improving the energy efficiency or the sum-rate. This problem can be formulated to optimize global energy efficiency of the system by jointly implementing the user clustering, APs association, and the power allocation.

\section{B. NOMA-VLC Networks}

Despite all the aforementioned work on NOMA-VLC systems, numerous challenges remain, and important topics in this area of research are still to be investigated. Below is a list of some key open problems in NOMA-VLC networks:

- Hybrid NOMA-VLC systems: the hybrid NOMA is to group the users into multiple clusters, and assign to each cluster a designated resource block, following the NOMA principle in each cluster. To our knowledge, the hybrid NOMA has not been studied yet in VLC systems. The rational for using the a hybrid NOMA is its ability to reduce the system's complexity. Indeed, having a large number of users in the VLC system, and assigning them to the same resource block can be problematic, since the user with the best channel must decode all the signals of all the users before decoding his/her own signal, creating delays the decoding and resulting in high complexity. Hybrid NOMA systems have been proposed in RF networks to take into account 
both the system performance and complexity. Therefore, we propose to study the hybrid NOMA-VLC system by finding the optimal user grouping, allocating the power to each group, the power inside each group, or grouping the users and allocating the power jointly, for sum-rate maximization purposes. This system can be extended to be a multi-cell system, in which the userto-AP association problem also exists and should be considered. Hence, the problem would be then a two layer user grouping with power allocation.

- NOMA with different QoS requirements: In real life, not all users require the same amount of data rate. For example, some of them may stream videos, whereas others are texting or exploring websites. Also some receivers can be IoT devices that need low data rates. Allocating power, in the most effective way, to users with different needs still remain a challenge, and obtaining the required data rate for each user, even when some weak users (users with poor channels) require higher data.

- Cooperative NOMA-VLC: cooperative NOMA has been proposed in RF networks to exploit the redundant information in NOMA systems, and to compensate the weak user suffering from co-channel interference by increasing his data rate. The cooperative NOMA can be used among VLC users or by using relays. We are interested in the cooperation among users, where the strong users that encode the signals of the weak users can forward these coded signals to the intended users. When assuming a VLC system with two users, under the assumption that all users are equipped with a multi-homing mechanism (i.e. they can gather information from VLC and RF networks simultaneously), the strong user first decodes the signal of the weak user, then forwards it to the weak user using WiFi or Bluetooth techniques. Weak user can then combine the RF and VLC signals, using combining techniques.

- Modulation and coding for NOMA-VLC system: several papers studied the modulation and coding schemes in NOMA RF networks [241], [242]. As the modulation and coding in VLC networks is different (based on IM/DD), investigating the modulation and coding schemes in NOMA-VLC systems would be worthy for practical implementation.

- NOMA in coordinated multi-point (CoMP) VLC networks: CoMP VLC system means that multiple APs are cooperating to transmit the data for the users. The cooperation is for mitigating the inter-cell interference and enhancing the received data rate by optimizing the precoding matrix. Assume a VLC system consisting of $N$ APs and $M$ users, where $M>N$, the questions should be raised is that how the users should be sorted from the strongest user to the weakest user?, how should the users be grouped to be served by the cooperating APs?, and how should the power be allocated. Combining the two techniques NOMA and CoMP surly leads to having a significant performance improvement in VLC systems.

- Hybrid SDMA and NOMA: SDMA in VLC can be implemented using angle diversity transmitters that can

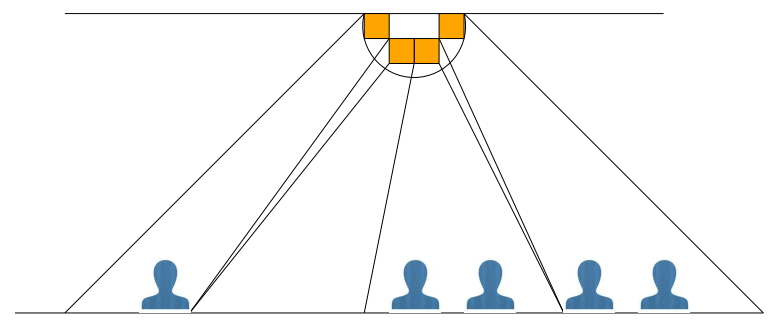

Fig. 11. The proposed hybrid SDMA/NOMA system.

generate several parallel narrow light beams directed to different directions using different LEDs. The goal of using SDMA is to mitigate inter-cell interference in VLC networks by directing the light to intended users and decreasing the overlap areas. However, some LEDs can be directed to non users, some to one user, and others to multiple users. The LEDs that are assigned or directed to serve multiple users can use the NOMA as a multiple access and to maximize the data rate. SDAM is used to mitigate or eliminate inter-cell interference, and NOMA is used to mitigate the intra-cell interference using SIC. By combining both of them, the system performance is significantly improved, in terms of data rate and system's fairness. Fig. 11 shows a system model in which SDMA and NOMA can coexist in VLC systems, where the NOMA can be used in LEDs that serve more than one user.

\section{Harvesting the Energy in VLC Systems}

Despite all the aforementioned work in Section VI, there are several remaining challenges associated with the transfer of information and power, using a light wave. Here are below a few key issues that need to be investigated and optimized for obtaining the most efficient power and information transfer systems.

- Simultaneous light-wave for information, illumination, and power transfer: Several studies investigated VLC systems in which both energy and information could be transferred to users. However, achieving both functions in VLC networks might violate the illumination requirements. We therefore propose to study the three functions of the light simultaneously by formulating optimization problems that allocate DC bias, available power, and available resources.

- Joint DC-bias and resource allocation for sum rate with the presence of energy-harvesting users: allocating both the DC bias and the available resources at the VLC APs leads to a significant improvement of the VLC performance under simultaneous lightwave information and power transfer (SLIPT). An effective allocation of resources (to the users) provides opportunities to preserve high energy that can be harvested by users.

- As proposed in the NOMA-VLC Section, a cooperative NOMA can be implemented in VLC systems; however, the strong user may do not want to consume some of his/her power by forwarding the signal to the weak user. We therefore suggest investigating ways for the strong 
user to harvest the energy from the light intensity, in the first phase, and then use it to forward the weak user's signal. This means that the transmitter should optimize the DC bias and the information power to maximize the sum rate and guarantee acceptable fairness.

- Optimizing the MISO-VLC network with NOMA: when the system consists of multiple VLC APs cooperating to transmit the information and power for multiple users, if the number of users is larger than the number of APs, the key issue that should be addressed is whether OMA or NOMA is the best system for scheduling users and harvesting the energy. As previously reported in the literature, NOMA can provide better data rates than OMA. In other words, NOMA can achieve the required users' data rate with a small amount of transmit power (information power), which allow the DC bias to increase, resulting in increasing the harvested energy.

- Placing the energy harvesting users: suppose that a VLC system consisting of multiple information users (users interested only in gathering the information), and IoT devices that work only in uplink (like sensors) and interested only in harvesting the energy (energy harvesting (EH) users). Optimizing the positions of the $\mathrm{EH}$ users in order to maximize the harvested energy and to achieve the required QoS at the information users is crucial in VLC SLIPT systems. Yet, it remains challenging. Therefore, there is needed to investigate ways of implementing and simplifying this task.

\section{Securing VLC Networks}

Despite the significant number of studies already performed, there are still some important issues to tackle and still many challenges for researchers to overcome in the future. A few of them are highlighted below, together with potential solutions that may improve the PLS in VLC systems:

- Joint PLS and load balancing in hybrid RF/VLC systems with considering the illumination constraints: All the conducted works on balancing the load in hybrid $\mathrm{VLC} / \mathrm{RF}$ networks are implemented to maximize the system utilities (such as sum-rate and/or fairness) without considering the secrecy constraints. Hence, we propose to design and optimize the joint load balancing with secrecy and illumination constraints when single or multiple, known or unknown, eavesdroppers exist. This problem contains joint user-to-APs association and power allocation to maximize the secrecy capacity and achieve the required illumination.

- How to optimize the beamforming vector in MISO-VLS systems when an active and passive eavesdropper exists. The common approach for the active eavesdropper is the zero-forcing preacoding approach, the common approach for the passive eavesdroppers is the design of protected zones, using an artificial noise or by steering the beamforming lobes. This raises an important question: what would the appropriate method be to improve the security, if the transmitters know the CSI of some eavesdroppers and they do not know the CSI of the others, or have a limited information about the eavesdropper (e.g. location only).

- PLS in NOMA-VLC system: Several recent papers investigated the PLS in NOMA RF networks for different system models [243], [244]. To this day, no paper has studied the PLS in NOMA-VLC systems. Because of the unique properties of VLC systems, the PLS in NOMAVLC systems is required to be investigated, evaluated, and optimized.

- User-centric cell formation based in the presence of eavesdroppers: As shown above, the user-centric cell formation is an appropriate scenario when the number of users is much smaller than the APs. Suppose that the network contains some eavesdroppers (whether their CSI are available or not), the questions raised are: 1) how should the users be clustered? 2) how should the APs be associated to the clustered users? 3) which APs should participate in communication, and which should be switched off? 4) could the switched off APs help enhance the secrecy sum-rate in emitting jamming signals?

All the above questions indicate that the joint PLS and user-centric design should be investigated and optimized together.

\section{E. Multi-User Outdoor VLC Networks}

Toward developing smart cities, the street and park lamps can be utilized as VLC APs that can be used to serve multiple users. The feasibility and popularity of the VLC outdoor communication depends highly on defeating or mitigating the effect of the contaminating light stemming from the sun and the ambient lights [245]. Most of the optimization techniques proposed for indoor VLC networks cannot be applied directly in outdoor VLC networks. To extend the techniques which have been proposed for indoor VLC networks to be used in outdoor VLC networks, different issues should be considered in formulating and solving the optimization problems:

- Because of the sun light, the receivers might be blinded to detect the received light since the ambient light illuminance might be stronger than the transmitter illuminance. This contaminating light varies during the day time and can be mitigated using lens and filter at the receivers [245]. Islim et al. [246] showed that in the presence of the sun light, a reliable communication can be achieved at high speed data rates and by employing an optical bandpass blue filter that can reduce the effect of the sun light.

- The outdoor VLC channel is not stable and static like the ones in the indoor. The outdoor VLC channel is attenuated due to the atmosphere pressure and the inhomogeneities in the temperature, resulting in having the refractive index varies along the transmission path [247].

- In outdoor VLC systems, the transmitters' distribution, transmitters' height, density of transmitters, and coverage area are different from those in indoor VLC systems [248]. In particular, the typical transmitters' height is 8 $\mathrm{m}$, the cell radius is around $7 \mathrm{~m}$, and the APs' distribution might be one dimensional in the street. Whereas, in 
indoor systems, the typical transmitters' height is $3 \mathrm{~m}$, the cell radius is around 2-3 m, and the APs' distribution is two dimensional in the ceiling. These outdoor features lead to having a less inter-cell interference (because of the one dimensional distribution), lower channels quality (because of the longer distances between users and receivers), larger cell coverage (because of the APs heights), and less rate of blockages than the case in indoor VLC systems. In addition, the required illumination (in Lux) in the outdoor is less restricted than the one in the indoor environments [248].

\section{ACKNOWLEDGMENT}

This work was supported by King Fahd University of Petroleum \& Minerals-King Abdullah University of Science and Technology (KFUPM-KAUST) initiative through grant number KAUST004.

\section{REFERENCES}

[1] "Cisco global cloud index: Forecast and methodology, 20162021 white paper," 2017.

[2] J. G. Andrews, S. Buzzi, W. Choi, S. V. Hanly, A. Lozano, A. C. Soong, and J. C. Zhang, "What will 5G be?" IEEE J. Sel. Areas Commun., vol. 32, no. 6, pp. 1065-1082, 2014.

[3] M. Shafi, A. F. Molisch, P. J. Smith, T. Haustein, P. Zhu, P. De Silva, F. Tufvesson, A. Benjebbour, and G. Wunder, "5G: A tutorial overview of standards, trials, challenges, deployment, and practice," IEEE J. Sel. Areas Commun., vol. 35, no. 6, pp. 1201-1221, 2017.

[4] P. Pirinen, "A brief overview of $5 \mathrm{~g}$ research activities," in 1st Int. Conf. $5 G$ for Ubiquitous Connectivity (5GU). IEEE, 2014, pp. 17-22.

[5] A. Al-Fuqaha, M. Guizani, M. Mohammadi, M. Aledhari, and M. Ayyash, "Internet of things: A survey on enabling technologies, protocols, and applications," IEEE Commun. Surveys Tuts., vol. 17, no. 4, pp. 2347-2376, 2015.

[6] M. R. Palattella, M. Dohler, A. Grieco, G. Rizzo, J. Torsner, T. Engel, and L. Ladid, "Internet of things in the 5G era: Enablers, architecture, and business models," IEEE J. Sel. Areas Commun., vol. 34, no. 3, pp. $510-527,2016$.

[7] D. Tsonev, S. Videv, and H. Haas, "Towards a $100 \mathrm{~Gb} / \mathrm{s}$ visible light wireless access network," Opt. Express, vol. 23, no. 2, pp. 1627-1637, 2015.

[8] N. Bhushan, J. Li, D. Malladi, R. Gilmore, D. Brenner, A. Damnjanovic, R. Sukhavasi, C. Patel, and S. Geirhofer, "Network densification: the dominant theme for wireless evolution into 5G," IEEE Commun. Mag., vol. 52, no. 2, pp. 82-89, 2014.

[9] X. Ge, S. Tu, G. Mao, C.-X. Wang, and T. Han, "5G ultra-dense cellular networks," IEEE Wireless Commun., vol. 23, no. 1, pp. 7279, 2016.

[10] Y. Tanaka, S. Haruyama, and M. Nakagawa, "Wireless optical transmissions with white colored LED for wireless home links," in Proc. 11th Symp. Pers. Indoor and Mobile Radio Commun (PIMRC), vol. 2. IEEE, 2000, pp. 1325-1329.

[11] T. Kishi, H. Tanaka, Y. Umeda, and O. Takyu, "A high-speed LED driver that sweeps out the remaining carriers for visible light communications," J. Lightw. Technol., vol. 32, no. 2, pp. 239-249, 2014.

[12] A. B. Siddique and M. Tahir, "Joint rate-brightness control using variable rate MPPM for LED based visible light communication systems," IEEE Trans. Wireless Commun., vol. 12, no. 9, pp. 46044611, 2013.

[13] J. M. Kahn and J. R. Barry, "Wireless infrared communications," Proc. IEEE, vol. 85, no. 2, pp. 265-298, 1997.

[14] M. Kavehrad, "Sustainable energy-efficient wireless applications using light," IEEE Commun. Mag., vol. 48, no. 12, pp. 66-73, 2010.

[15] H. Elgala, R. Mesleh, and H. Haas, "Indoor optical wireless communication: potential and state-of-the-art," IEEE Commun. Mag., vol. 49, no. 9, 2011.

[16] I. Din and H. Kim, "Energy-efficient brightness control and data transmission for visible light communication," IEEE Photon. Technol. Lett., vol. 26, no. 8, pp. 781-784, 2014.
[17] S. Dimitrov and H. Haas, "Information rate of OFDM-based optical wireless communication systems with nonlinear distortion," J. Lightw. Technol., vol. 31, no. 6, pp. 918-929, 2013.

[18] _ Principles of LED light communications: towards networked Li-Fi. Cambridge University Press, 2015.

[19] J. Miyakoshi, "Cellular and molecular responses to radio-frequency electromagnetic fields," Proc. IEEE, vol. 101, no. 6, pp. 1494-1502, 2013.

[20] L. Feng, R. Q. Hu, J. Wang, P. Xu, and Y. Qian, "Applying VLC in 5G networks: Architectures and key technologies," IEEE Netw., vol. 30, no. 6, pp. 77-83, 2016.

[21] N. Kumar and N. R. Lourenco, "LED-based visible light communication system: a brief survey and investigation," J. Eng. Appl. Sci, vol. 5, no. 4, pp. 296-307, 2010.

[22] A. Sevincer, A. Bhattarai, M. Bilgi, M. Yuksel, and N. Pala, "Lightnets: Smart LIGHTing and mobile optical wireless NETworksA survey," IEEE Commun. Surveys Tuts., vol. 15, no. 4, pp. 1620-1641, 2013.

[23] S. Wu, H. Wang, and C.-H. Youn, "Visible light communications for $5 \mathrm{G}$ wireless networking systems: from fixed to mobile communications," IEEE Netw., vol. 28, no. 6, pp. 41-45, 2014.

[24] D. Karunatilaka, F. Zafar, V. Kalavally, and R. Parthiban, "LED based indoor visible light communications: State of the art." IEEE Commun. Surveys Tuts., vol. 17, no. 3, pp. 1649-1678, 2015.

[25] P. H. Pathak, X. Feng, P. Hu, and P. Mohapatra, "Visible light communication, networking, and sensing: A survey, potential and challenges," IEEE Commun. Surveys Tuts., vol. 17, no. 4, pp. 20472077, 2015.

[26] X. Li, R. Zhang, and L. Hanzo, "Optimization of visible-light optical wireless systems: Network-centric versus user-centric designs," IEEE Commun. Surveys Tuts., 2018.

[27] M. Z. Chowdhury, M. T. Hossan, A. Islam, and Y. M. Jang, "A comparative survey of optical wireless technologies: architectures and applications," IEEE Access, vol. 6, pp. 9819-9840, 2018.

[28] Y. Qiu, H.-H. Chen, and W.-X. Meng, "Channel modeling for visible light communicationsa survey," Wireless Communications and Mobile Computing, vol. 16, no. 14, 2016.

[29] K. Sindhubala and B. Vijayalakshmi, "Survey on noise sources and restrain techniques in visible-light communication." Light \& Engineering, vol. 24, no. 2, 2016.

[30] Y. Zhuang, L. Hua, L. Qi, J. Yang, P. Cao, Y. Cao, Y. Wu, J. Thompson, and $\mathrm{H}$. Haas, "A survey of positioning systems using visible LED lights," IEEE Commun. Surveys Tuts., 2018.

[31] A.-M. Căilean and M. Dimian, "Current challenges for visible light communications usage in vehicle applications: A survey," IEEE Commun. Surveys Tuts., vol. 19, no. 4, pp. 2681-2703, 2017.

[32] A. Khalid, G. Cossu, R. Corsini, P. Choudhury, and E. Ciaramella, "1-Gb/s transmission over a phosphorescent white LED by using rateadaptive discrete multitone modulation," IEEE Photon. J., vol. 4, no. 5, pp. 1465-1473, 2012.

[33] G. Cossu, A. Khalid, P. Choudhury, R. Corsini, and E. Ciaramella, "3.4 gbit/s visible optical wireless transmission based on RGB LED," Opt. Express, vol. 20, no. 26, pp. B501-B506, 2012.

[34] Z. Wang, D. Tsonev, S. Videv, and H. Haas, "On the design of a solarpanel receiver for optical wireless communications with simultaneous energy harvesting," IEEE J. Sel. Areas Commun., vol. 33, no. 8, pp. 1612-1623, 2015.

[35] T. Komine and M. Nakagawa, "Fundamental analysis for visiblelight communication system using LED lights," IEEE Trans. Consum. Electron., vol. 50, no. 1, pp. 100-107, 2004.

[36] F. R. Gfeller and U. Bapst, "Wireless in-house data communication via diffuse infrared radiation," Proc. IEEE, vol. 67, no. 11, pp. 1474-1486, 1979.

[37] A. Al-Kinani, C.-X. Wang, L. Zhou, and W. Zhang, "Optical wireless communication channel measurements and models," IEEE Commun. Surveys Tuts., vol. 20, no. 3, pp. 1939-1962, 2018.

[38] M. D. Soltani, X. Wu, M. Safari, and H. Haas, "Access point selection in Li-Fi cellular networks with arbitrary receiver orientation," in Proc. 27th Annual Int. Symp. Pers. Indoor, and Mobile Radio Commun. (PIMRC). IEEE, 2016, pp. 1-6.

[39] M. D. Soltani, A. A. Purwita, Z. Zeng, H. Haas, and M. Safari, "Modeling the random orientation of mobile devices: Measurement, analysis and LiFi use case," IEEE Trans. Commun., pp. 1-1, 2018.

[40] Y. S. Eroglu, Y. Yapici, and I. Guvenc, "Impact of random receiver orientation on visible light communications channel," IEEE Trans. Commun., pp. 1-1, 2018. 
[41] Q. Wang, Z. Wang, and L. Dai, "Multiuser MIMO-OFDM for visible light communications," IEEE Photon. J., vol. 7, no. 6, pp. 1-11, 2015.

[42] A. Yesilkaya, F. Miramirkhani, E. Basar, E. Panayirci, and M. Uysal, "Performance of mimo enhanced unipolar ofdm with realistic indoor visible light channel models," in Proc. Wireless Commun. and Netw. Conf. (WCNC). IEEE, 2016, pp. 1-6.

[43] S. Rajagopal, R. D. Roberts, and S.-K. Lim, "Ieee 802.15. 7 visible light communication: modulation schemes and dimming support," IEEE Commun. Mag., vol. 50, no. 3, 2012.

[44] H. Le Minh, D. O’Brien, G. Faulkner, L. Zeng, K. Lee, D. Jung, Y. Oh, and E. T. Won, "100-mb/s nrz visible light communications using a postequalized white LED," IEEE Photon. Technol. Lett., vol. 21, no. 15, pp. 1063-1065, 2009.

[45] G. Ntogari, T. Kamalakis, J. Walewski, and T. Sphicopoulos, "Combining illumination dimming based on pulse-width modulation with visible-light communications based on discrete multitone," IEEE J. Opt. Commun. Netw., vol. 3, no. 1, pp. 56-65, 2011.

[46] B. Bai, Z. Xu, and Y. Fan, "Joint LED dimming and high capacity visible light communication by overlapping PPM," in 19th Annu. Wireless and Optical Commun. Conf. (WOCC). IEEE, 2010, pp. $1-5$.

[47] H. Sugiyama and K. Nosu, "MPPM: A method for improving the band-utilization efficiency in optical PPM," J. Lightw. Technol., vol. 7, no. 3, pp. 465-472, 1989.

[48] T. Ohtsuki, I. Sasase, and S. Mori, "Overlapping multi-pulse pulse position modulation in optical direct detection channel," in Proc. Int. Conf. Commun. Technical Program, Conf. Record, ICC'93 Geneva, vol. 2. IEEE, 1993, pp. 1123-1127.

[49] H. Elgala, R. Mesleh, H. Haas, and B. Pricope, "OFDM visible light wireless communication based on white LEDs," in IEEE 65th Veh. Technol. Conf. (VTC2007)-Spring. IEEE, 2007, pp. 2185-2189.

[50] M. Z. Afgani, H. Haas, H. Elgala, and D. Knipp, "Visible light communication using OFDM," in 2nd Int. Conf. Testbeds and Research Infrastructures for the Development of Networks and Communities, TRIDENTCOM. IEEE, 2006, pp. 6-pp.

[51] J. Armstrong and A. Lowery, "Power efficient optical OFDM," Electron. Lett., vol. 42, no. 6, pp. 370-372, 2006.

[52] A. Lapidoth, S. M. Moser, and M. A. Wigger, "On the capacity of free-space optical intensity channels," IEEE Trans. Inf. Theory, vol. 55, no. 10 , pp. 4449-4461, 2009.

[53] J. G. Smith, "The information capacity of amplitude-and varianceconstrained sclar gaussian channels," Information and Control, vol. 18, no. 3, pp. 203-219, 1971

[54] T. H. Chan, S. Hranilovic, and F. R. Kschischang, "Capacity-achieving probability measure for conditionally gaussian channels with bounded inputs," IEEE Trans. Inf. Theory, vol. 51, no. 6, pp. 2073-2088, 2005.

[55] B. Mamandipoor, K. Moshksar, and A. K. Khandani, "Capacityachieving distributions in gaussian multiple access channel with peak power constraints," IEEE Trans. Inf. Theory, vol. 60, no. 10, pp. 60806092, 2014.

[56] A. ElMoslimany and T. M. Duman, "On the discreteness of capacityachieving distributions for fading and signal-dependent noise channels with amplitude-limited inputs," IEEE Trans. Inf. Theory, vol. 64, no. 2, pp. 1163-1177, 2018.

[57] M. Soltani and Z. Rezki, "The capacity of the optical broadcast channel with peak and average intensity constraints," University of Idaho, ID, Tech. Rep, 2018.

[58] A. A. Farid and S. Hranilovic, "Channel capacity and non-uniform signalling for free-space optical intensity channels," IEEE J. Sel. Area. Commun., vol. 27, no. 9, 2009.

[59] A. Chaaban, J.-M. Morvan, and M.-S. Alouini, "Free-space optical communications: Capacity bounds, approximations, and a new spherepacking perspective," IEEE Trans. Commun., vol. 64, no. 3, pp. 11761191, 2016.

[60] K.-I. Ahn and J. K. Kwon, "Capacity analysis of M-PAM inverse source coding in visible light communications," J. Lightw. Technol., vol. 30, no. 10, pp. 1399-1404, 2012.

[61] J.-B. Wang, Q.-S. Hu, J. Wang, M. Chen, and J.-Y. Wang, "Tight bounds on channel capacity for dimmable visible light communications," J. Lightw. Technol., vol. 31, no. 23, pp. 3771-3779, 2013.

[62] J.-B. Wang, Q.-S. Hu, J. Wang, M. Chen, Y.-H. Huang, and J.-Y. Wang, "Capacity analysis for dimmable visible light communications," in Proc. Int. Conf. Commun. (ICC). IEEE, 2014, pp. 3331-3335.

[63] R. Jiang, Z. Wang, Q. Wang, and L. Dai, "A tight upper bound on channel capacity for visible light communications," IEEE Commun. Lett., vol. 20, no. 1, pp. 97-100, 2016
[64] A. Chaaban, Z. Rezki, and M.-S. Alouini, "On the capacity of the intensity-modulation direct-detection optical broadcast channel," IEEE Trans. Wireless Commun., vol. 15, no. 5, pp. 3114-3130, 2016.

[65] K. Xu, H.-Y. Yu, Y.-J. Zhu, and Y. Sun, "On the ergodic channel capacity for indoor visible light communication systems," IEEE Access, vol. 5, pp. 833-841, 2017.

[66] J.-Y. Wang, Q.-L. Li, J.-X. Zhu, and Y. Wang, "Impact of receiver's tilted angle on channel capacity in vlcs," Electron. Lett., vol. 53, no. 6, pp. 421-423, 2017.

[67] S. M. Moser, L. Wang, and M. Wigger, "Asymptotic high-snr capacity of miso optical intensity channels," in IEEE Inform. Theory Workshop (ITW). IEEE, 2017, pp. 86-90.

[68] S. M. Moser, M. Mylonakis, L. Wang, and M. Wigger, "Asymptotic capacity results for mimo wireless optical communication," in IEEE Int. Symp. Information Theory (ISIT). IEEE, 2017, pp. 536-540.

[69] S. M. Moser, L. Wang, and M. Wigger, "Capacity results on multipleinput single-output wireless optical channels," IEEE Trans. Inf. Theory, 2018

[70] A. Chaaban, Z. Rezki, and M.-S. Alouini, "Low-snr asymptotic capacity of mimo optical intensity channels with peak and average constraints," IEEE Trans. Commun., 2018.

[71] _ _ "Capacity bounds and high-snr capacity of mimo intensitymodulation optical channels," IEEE Trans. Wireless Commun., vol. 17, no. 5, pp. 3003-3017, 2018.

[72] A. Agarwal and S. K. Mohammed, "Achievable rate region of the zero-forcing precoder in a $2 \times 2$ MU-MISO broadcast VLC channel with per-LED peak power constraint and dimming control," J. Lightw. Technol., vol. 35, no. 19, pp. 4168-4194, 2017.

[73] Y. Liang, H. V. Poor, S. Shamai et al., "Information theoretic security," Foundations and Trends $\AA$ in Communications and Information Theory, vol. 5, no. 4-5, pp. 355-580, 2009.

[74] O. Ozel, E. Ekrem, and S. Ulukus, "Gaussian wiretap channel with amplitude and variance constraints," IEEE Trans. Inf. Theory, vol. 61, no. 10, pp. 5553-5563, 2015.

[75] Z. Rezki and M.-S. Alouini, "Secret-key agreement with public discussion over multi-antenna transmitters with amplitude constraints," in IEEE Int. Symp. Information Theory (ISIT). IEEE, 2017, pp. 15341538.

[76] A. Mostafa and L. Lampe, "Securing visible light communications via friendly jamming," in Proc. Global Commun. Conf. (Globecom). IEEE, 2014, pp. 524-529.

[77] H. Zaid, Z. Rezki, A. Chaaban, and M. S. Alouini, "Improved achievable secrecy rate of visible light communication with cooperative jamming," in Global Conf. Signal and Inform. Processing (GlobalSIP). IEEE, 2015, pp. 1165-1169.

[78] A. Mostafa and L. Lampe, "Pattern synthesis of massive LED arrays for secure visible light communication links," in Int. Conf. Commun. Workshop (ICCW). IEEE, 2015, pp. 1350-1355.

[79] M. A. Arfaoui, Z. Rezki, A. Ghrayeb, and M. S. Alouini, "On the secrecy capacity of MISO visible light communication channels," in Proc. Global Commun. Conf. (Globecom). IEEE, 2016, pp. 1-7.

[80] _ - "On the input distribution and optimal beamforming for the MISO VLC wiretap channel," in Global Conf. Signal and Inform. Processing (GlobalSIP). IEEE, 2016, pp. 970-974.

[81] A. Mostafa and L. Lampe, "Physical-layer security for MISO visible light communication channels," IEEE J. Sel. Areas Commun., vol. 33, no. 9, pp. 1806-1818, 2015.

[82] M. A. Arfaoui, Z. Rezki, A. Ghrayeb, and M. S. Alouini, "Discrete input signaling for MISO visible light communication channels," in Proc. Wireless Commun. and Netw. Conf. (WCNC). IEEE, 2017, pp. $1-6$.

[83] M. A. Arfaoui, A. Ghrayeb, and C. Assi, "Secrecy rate closedform expressions for the siso vlc wiretap channel with discrete input signaling," IEEE Commun. Lett., 2018.

[84] D. Tsonev, H. Chun, S. Rajbhandari, J. J. McKendry, S. Videv, E. Gu, M. Haji, S. Watson, A. E. Kelly, G. Faulkner et al., "A 3-gb/s singleled ofdm-based wireless vlc link using a gallium nitride $\mu$ led," IEEE Photon. Technol. Lett., vol. 26, no. 7, pp. 637-640, 2014.

[85] Y. Wang, X. Wu, and H. Haas, "Load balancing game with shadowing effect for indoor hybrid LiFi/RF networks," IEEE Trans. Wireless Commun., vol. 16, no. 4, pp. 2366-2378, Apr. 2017.

[86] H. Burchardt, S. Sinanovic, Z. Bharucha, and H. Haas, "Distributed and autonomous resource and power allocation for wireless networks," IEEE Trans. Commun., vol. 61, no. 7, pp. 2758-2771, 2013.

[87] S. Buzzi, I. Chih-Lin, T. E. Klein, H. V. Poor, C. Yang, and A. Zappone, "A survey of energy-efficient techniques for $5 \mathrm{G}$ networks and 
challenges ahead," IEEE J. Sel. Areas Commun., vol. 34, no. 4, pp. 697-709, 2016.

[88] R. Jain, D.-M. Chiu, and W. R. Hawe, A quantitative measure of fairness and discrimination for resource allocation in shared computer system. Eastern Research Laboratory, Digital Equipment Corporation Hudson, MA, 1984, vol. 38.

[89] L. Li, M. Pal, and Y. R. Yang, "Proportional fairness in multi-rate wireless LANs," in IEEE 27th Conf. Computer Commun. (INFOCOM). IEEE, 2008, pp. 1004-1012.

[90] M. Uchida and J. Kurose, "An information-theoretic characterization of weighted alpha-proportional fairness," in IEEE INFOCOM. IEEE, 2009, pp. 1053-1061.

[91] R. Zhang, H. Claussen, H. Haas, and L. Hanzo, "Energy efficient visible light communications relying on amorphous cells," IEEE $J$. Sel. Areas Commun., vol. 34, no. 4, pp. 894-906, 2016.

[92] J. Grubor, S. Randel, K.-D. Langer, and J. W. Walewski, "Broadband information broadcasting using LED-based interior lighting," $J$. Lightw. Technol., vol. 26, no. 24, pp. 3883-3892, 2008.

[93] S. Dimitrov, S. Sinanovic, and H. Haas, "Clipping noise in OFDMbased optical wireless communication systems," IEEE Trans. Commun., vol. 60, no. 4, pp. 1072-1081, 2012.

[94] J. Gancarz, H. Elgala, and T. D. Little, "Impact of lighting requirements on VLC systems," IEEE Commun. Mag., vol. 51, no. 12, pp. 34-41, 2013.

[95] S. Lou, C. Gong, N. Wu, and Z. Xu, "Joint dimming and communication design for visible light communication," IEEE Commun. Lett., vol. 21, no. 5, pp. 1043-1046, 2017.

[96] K. Lee and H. Park, "Modulations for visible light communications with dimming control," IEEE Photon. Technol. Lett., vol. 23, no. 16, pp. 1136-1138, 2011

[97] "IEEE standard for local and metropolitan area networkspart 15.7: Short-range wireless optical communication using visible light," IEEE Std 802.15.7.2011, pp. 1-309, Sep. 2011.

[98] C. Chen, S. Videv, D. Tsonev, and H. Haas, "Fractional frequency reuse in DCO-OFDM-based optical attocell networks," J. Lightw. Technol., vol. 33, no. 19, pp. 3986-4000, 2015.

[99] C. Li, W. Jia, Q. Tao, and M. Sun, "Solar cell phone charger performance in indoor environment," in IEEE 37th Annu. Northeast Bioengineering Conference (NEBEC). IEEE, 2011, pp. 1-2.

[100] J. G. Andrews, S. Singh, Q. Ye, X. Lin, and H. S. Dhillon, "An overview of load balancing in hetnets: Old myths and open problems," IEEE Wireless Commun., vol. 21, no. 2, pp. 18-25, 2014.

[101] E. Aryafar, A. Keshavarz-Haddad, M. Wang, and M. Chiang, "RAT selection games in HetNets," in Proc. IEEE INFOCOM. IEEE, 2013, pp. $998-1006$

[102] Y. Wang and H. Haas, "Dynamic load balancing with handover in hybrid Li-Fi and Wi-Fi networks," J. Lightw. Technol., vol. 33, no. 22, pp. 4671-4682, Nov. 2015.

[103] L. Li, Y. Zhang, B. Fan, and H. Tian, "Mobility-aware load balancing scheme in hybrid vlc-lte networks," IEEE Commun. Lett., vol. 20, no. 11, pp. 2276-2279, 2016

[104] Y. Wang, D. A. Basnayaka, and H. Haas, "Dynamic load balancing for hybrid Li-Fi and RF indoor networks," in IEEE Int. Conf. Commun. Workshops (ICCW), London, UK, 2015, pp. 1422-1427.

[105] M. Kassab, J. M. Bonnin, and A. Belghith, "Fast and secure handover in WLANs: An evaluation of the signaling overhead," in Consumer Commun. and Netw. Conf. (CCNC) 5th IEEE. IEEE, 2008, pp. 770775.

[106] H. Haas, L. Yin, Y. Wang, and C. Chen, "What is lifi?" J. Lightw. Technol., vol. 34, no. 6, pp. 1533-1544, 2016.

[107] M. Ayyash, H. Elgala, A. Khreishah, V. Jungnickel, T. Little, S. Shao, M. Rahaim, D. Schulz, J. Hilt, and R. Freund, "Coexistence of WiFi and LiFi toward 5G: concepts, opportunities, and challenges," IEEE Commun. Mag., vol. 54, no. 2, pp. 64-71, 2016.

[108] X. Wu, M. Safari, and H. Haas, "Access point selection for hybrid Li-Fi and Wi-Fi networks," IEEE Trans. Commun., vol. 65, no. 12, pp. 5375-5385, 2017.

[109] I. Stefan and H. Haas, "Hybrid visible light and radio frequency communication systems," in IEEE 80th Veh. Technol. Conf. (VTC Fall). IEEE, 2014, pp. 1-5.

[110] D. A. Basnayaka and H. Haas, "Hybrid RF and VLC systems: Improving user data rate performance of VLC systems," in Proc. IEEE Veh. Technol. Conf. (VTC Spring), Glasgow, UK, May 2015, pp. 1-5.

[111] X. Li, R. Zhang, and L. Hanzo, "Cooperative load balancing in hybrid visible light communications and WiFi," IEEE Trans. Commun., vol. 63, no. 4, pp. 1319-1329, 2015.
[112] Y. Wang, D. A. Basnayaka, X. Wu, and H. Haas, "Optimization of load balancing in hybrid LiFi/RF networks," IEEE Trans. Commun., vol. 65 , no. 4, pp. 1708-1720, 2017.

[113] X. Wu, M. Safari, and H. Haas, "Joint optimisation of load balancing and handover for hybrid LiFi and WiFi networks," in Proc. Wireless Commun. and Netw. Conf. (WCNC). San Francisco, USA: IEEE, 2017, pp. 1-5.

[114] X. Wu and H. Haas, "Access point assignment in hybrid LiFi and WiFi networks in consideration of LiFi channel blockage," in IEEE 18th Int. Workshop Signal Processing Advances in Wireless Commun. (SPAWC). IEEE, 2017, pp. 1-5.

[115] X. Wu, D. Basnayaka, M. Safari, and H. Haas, "Two-stage access point selection for hybrid VLC and RF networks," in Proc. 27th Annual Int. Symp. Pers. Indoor, and Mobile Radio Commun. (PIMRC). IEEE, 2016, pp. 1-6.

[116] Y. Wang, X. Wu, and H. Haas, "Fuzzy logic based dynamic handover scheme for indoor Li-Fi and RF hybrid network," in Proc. Int. Conf. Commun. (ICC). IEEE, 2016, pp. 1-6.

[117] Y. Wang and H. Haas, "A comparison of load balancing techniques for hybrid LiFi/RF networks," in Proc. 4th ACM Workshop on Visible Light Commun. Syst. ACM, 2017, pp. 43-47.

[118] J. Wang, C. Jiang, H. Zhang, X. Zhang, V. C. Leung, and L. Hanzo, "Learning-aided network association for hybrid indoor LiFi-WiFi systems," IEEE Trans. Veh. Technol., 2017.

[119] M. Obeed, A. M. Salhab, S. A. Zummo, and M.-S. Alouini, "Joint load balancing and power allocation for hybrid VLC/RF networks," in Proc. IEEE Global Commun. Conf. (Globecom), Singapore, 2017.

[120] —, "Joint optimization of power allocation and load balancing for hybrid VLC/RF networks," IEEE/OSA J. Opt. Commun. and Netw., 2018.

[121] F. Kelly, "Charging and rate control for elastic traffic," Trans. Emerg. Telecommun. Technol., vol. 8, no. 1, pp. 33-37, 1997.

[122] F. Jin, R. Zhang, and L. Hanzo, "Resource allocation under delayguarantee constraints for heterogeneous visible-light and RF femtocell," IEEE Trans. Wireless Commun., vol. 14, no. 2, pp. 1020-1034, 2015.

[123] M. Kafafy, Y. Fahmy, M. Abdallah, and M. Khairy, "A novel bandwidth and power allocation scheme for power efficient hybrid RF/VLC indoor systems," Physical Communication, 2018.

[124] M. Kashef, M. Ismail, M. Abdallah, K. A. Qaraqe, and E. Serpedin, "Energy efficient resource allocation for mixed RF/VLC heterogeneous wireless networks," IEEE J. Sel. Areas Commun., vol. 34, no. 4, pp. 883-893, 2016.

[125] M. Kafafy, Y. Fahmy, M. Abdallah, and M. Khairy, "Power efficient downlink resource allocation for hybrid RF/VLC wireless networks," in Proc. Wireless Commun. and Netw. Conf. (WCNC). IEEE, 2017, pp. 1-6.

[126] M. Kashef, M. Abdallah, and N. Al-Dhahir, "Transmit power optimization for a hybrid PLC/VLC/RF communication system," IEEE Transactions on Green Communications and Networking, 2017.

[127] H. Zhang, N. Liu, K. Long, J. Cheng, V. C. M. Leung, and L. Hanzo, "Energy efficient subchannel and power allocation for the software defined heterogeneous VLC and RF networks," IEEE J. Sel. Areas Commun., vol. PP, no. 99, pp. 1-1, 2018.

[128] D. A. Basnayaka and H. Haas, "Design and analysis of a hybrid radio frequency and visible light communication system," IEEE Trans. Commun., vol. 65 , no. 10 , pp. 4334-4347, 2017.

[129] H. Tabassum and E. Hossain, "Coverage and rate analysis for coexisting rf/vlc downlink cellular networks," IEEE Trans. Wireless Commun., 2018.

[130] M. Hammouda, S. Akin, A. M. Vegni, H. Haas, and J. Peissig, "Link selection in hybrid RF/VLC systems under statistical queueing constraints," IEEE Trans. Wireless Commun., 2018.

[131] X. Wu, M. Safari, and H. Haas, "Bidirectional allocation game in visible light communications," in Proc. IEEE Veh. Technol. Conf. (VTC Spring), Montreal, Canada, 2016, pp. 1-5.

[132] R. Zhang, Y. Cui, H. Claussen, H. Haas, and L. Hanzo, "Anticipatory association for indoor visible light communications: Light, follow me!" IEEE Trans. Wireless Commun., 2018.

[133] R. Jiang, Q. Wang, H. Haas, and Z. Wang, "Joint user association and power allocation for cell-free visible light communication networks," IEEE J. Sel. Areas Commun., 2017.

[134] C. Chen, D. A. Basnayaka, and H. Haas, "Downlink performance of optical attocell networks," J. Lightw. Technol., vol. 34, no. 1, pp. 137156, 2016.

[135] Y. Wang, X. Wu, and H. Haas, "Resource allocation in LiFi OFDMA 
systems," in Proc. Global Commun. Conf. (Globecom), Dec 2017, pp. $1-6$.

[136] X. Ling, J. Wang, Z. Ding, C. Zhao, and X. Gao, "Efficient OFDMA for LiFi downlink," J. Lightw. Technol., 2018.

[137] L. Chen, W. Wang, and C. Zhang, "Coalition formation for interference management in visible light communication networks," IEEE Trans. Veh. Technol., vol. 66, no. 8, pp. 7278-7285, 2017.

[138] C. Chen, D. Tsonev, and H. Haas, "Joint transmission in indoor visible light communication downlink cellular networks," in Proc. Global Commun. Conf. (Globecom). IEEE, 2013, pp. 1127-1132.

[139] C. Chen and H. Haas, "Performance evaluation of downlink cooperative multipoint joint transmission in LiFi systems," in Proc. Globecom Workshops (GC Wkshps). IEEE, 2017, pp. 1-6.

[140] M. Kashef, M. Abdallah, K. Qaraqe, H. Haas, and M. Uysal, "Coordinated interference management for visible light communication systems," IEEE J. Opt. Commun. Netw., vol. 7, no. 11, pp. 1098-1108, 2015.

[141] C. Chen, N. Serafimovski, and H. Haas, "Fractional frequency reuse in optical wireless cellular networks," in Proc. 24th Int. Symp. Pers. Indoor and Mobile Radio Commun. (PIMRC). IEEE, 2013, pp. 3594 3598.

[142] Z.-G. Sun, H. Yu, and Y.-J. Zhu, "Efficient signal design and optimal power allocation for visible light communication attocell systems," Appl. Opt., vol. 56, no. 32, pp. 8959-8968, 2017.

[143] H. Ma, A. Mostafa, L. Lampe, and S. Hranilovic, "Coordinated beamforming for downlink visible light communication networks," IEEE Trans. Commun., 2018.

[144] A. Burton, Z. Ghassemlooy, S. Rajbhandari, and S.-K. Liaw, "Design and analysis of an angular-segmented full-mobility visible light communications receiver," Trans. Emerg. Telecommun. Technol., vol. 25, no. 6, pp. 591-599, mar 2014.

[145] A. Nuwanpriya, S.-W. Ho, and C. S. Chen, "Indoor MIMO visible light communications: Novel angle diversity receivers for mobile users," IEEE J. Sel. Areas Commun., vol. 33, no. 9, pp. 1780-1792, sep 2015.

[146] C. Chen, W.-D. Zhong, and D. Wu, "On the coverage of multiple-input multiple-output visible light communications [invited]," IEEE J. Opt. Commun. Netw., vol. 9, no. 9, p. D31, aug 2017.

[147] Z. Chen, D. A. Basnayaka, X. Wu, and H. Haas, "Interference mitigation for indoor optical attocell networks using an angle diversity receiver," J. Lightwave Technol., vol. 36, no. 18, pp. 3866-3881, sep 2018.

[148] K.-H. Park, H. M. Oubei, W. G. Alheadary, B. S. Ooi, and M.-S Alouini, "A novel mirror-aided non-imaging receiver for indoor $2 \times$ 2 mimo-visible light communication systems," IEEE Trans. Wireless Commun., vol. 16, no. 9, pp. 5630-5643, 2017.

[149] Z. Yu, R. J. Baxley, and G. T. Zhou, "Multi-user MISO broadcasting for indoor visible light communication," in IEEE Int. Conf. Acoustics, Speech and Signal Processing (ICASSP). IEEE, 2013, pp. 4849-4853.

[150] H. Ma, L. Lampe, and S. Hranilovic, "Robust MMSE linear precoding for visible light communication broadcasting systems," in Proc. Global Commun. Conf. (Globecom). IEEE, 2013, pp. 1081-1086.

[151] B. Li, J. Wang, R. Zhang, H. Shen, C. Zhao, and L. Hanzo, "Multiuser MISO transceiver design for indoor downlink visible light communication under per-LED optical power constraints," IEEE Photon. J., vol. 7 , no. 4 , pp. $1-15,2015$.

[152] H. Ma, L. Lampe, and S. Hranilovic, "Coordinated broadcasting for multiuser indoor visible light communication systems," IEEE Trans. Commun., vol. 63, no. 9, pp. 3313-3324, 2015.

[153] H. Shen, Y. Deng, W. Xu, and C. Zhao, "Rate-maximized zero-forcing beamforming for VLC multiuser MISO downlinks," IEEE Photon. J., vol. 8, no. 1, pp. 1-13, 2016.

[154] T. V. Pham, H. Le-Minh, and A. T. Pham, "Multi-user visible light communication broadcast channels with zero-forcing precoding," IEEE Trans. Commun., vol. 65, no. 6, pp. 2509-2521, 2017.

[155] Y. Hong, J. Chen, Z. Wang, and C. Yu, "Performance of a precoding mimo system for decentralized multiuser indoor visible light communications," IEEE Photon. J., vol. 5, no. 4, pp. 7800211-7800 211, 2013.

[156] T. V. Pham, H. Le Minh, Z. Ghassemlooy, T. Hayashi, and A. T. Pham, "Sum-rate maximization of multi-user MIMO visible light communications," in Proc. Int. Conf. Commun. Workshop (ICCW). IEEE, 2015, pp. 1344-1349.

[157] J. Chen, N. Ma, Y. Hong, and C. Yu, "On the performance of MUMIMO indoor visible light communication system based on THP algorithm," in IEEE/CIC Int. Conf. Commun. in China (ICCC). IEEE, 2014, pp. 136-140.
[158] H. Sifaou, A. Kammoun, K.-H. Park, and M.-S. Alouini, "Robust transceivers design for multi-stream multi-user MIMO visible light communication," IEEE Access, vol. 5, pp. 26387-26399, 2017.

[159] H. Marshoud, D. Dawoud, V. M. Kapinas, G. K. Karagiannidis, S. Muhaidat, and B. Sharif, "MU-MIMO precoding for VLC with imperfect CSI," in 4th Int. Workshop Opt. Wireless Commun. (IWOW). IEEE, 2015, pp. 93-97.

[160] K. Cai, M. Jiang, and X. Ma, "Photodetector selection aided multiuser MIMO optical OFDM imaging visible light communication system," IEEE Access, vol. 4, pp. 9870-9879, 2016.

[161] H. Marshoud, P. C. Sofotasios, S. Muhaidat, B. S. Sharif, and G. K. Karagiannidis, "Optical adaptive precoding for visible light communications," IEEE Access, 2018.

[162] C. Quintana, H. Chun, R. Mulyawan, G. Faulkner, S. Collins, D. C. O. Brien, E. Xie, J. J. D. McKendry, E. Gu, M. D. Dawson, P. P. Manousiadis, D. A. Vithanage, G. A. Turnbull, and I. D. Samuel, "Ultra-wide coverage VLC system with alignment-free receiver," in Global LIFI Congr. (GLC). IEEE, feb 2018.

[163] M. Obeed, A. M. Salhab, S. A. Zummo, and M.-S. Alouini, "New algorithms for energy-efficient VLC networks with user-centric cell formation," IEEE Trans. Green Commun. and Netw., 2018.

[164] Z. Chen and H. Haas, "Space division multiple access in visible light communications," in Proc. Int. Conf. Commun. (ICC). IEEE, 2015, pp. $5115-5119$.

[165] Z. Chen, D. A. Basnayaka, and H. Haas, "Space division multiple access for optical attocell network using angle diversity transmitters," J. Lightw. Technol., vol. 35, no. 11, pp. 2118-2131, 2017.

[166] Y. S. Eroğlu, İ. Güvenç, A. Şahin, Y. Yapıcı, N. Pala, and M. Yüksel, "Multi-element VLC networks: LED assignment, power control, and optimum combining," IEEE J. Sel. Areas Commun., vol. 36, no. 1, pp. 121-135, 2018.

[167] H. Marshoud, S. Muhaidat, P. C. Sofotasios, S. Hussain, M. A. Imran, and B. S. Sharif, "Optical non-orthogonal multiple access for visible light communication," IEEE Wireless Commun., vol. 25, no. 2, pp. 82-88, apr 2018.

[168] S.-M. Kim and H.-J. Lee, "Visible light communication based on space-division multiple access optical beamforming," Chinese Optics Lett., vol. 12, no. 12, pp. 120601-120601, 2014.

[169] L. Yin, X. Wu, H. Haas, and L. Hanzo, "Low-complexity SDMA usergrouping for the CoMP-VLC downlink," in Proc. Global Commun. Conf. (Globecom). IEEE, 2015, pp. 1-6.

[170] R. Zhang, J. Wang, Z. Wang, Z. Xu, C. Zhao, and L. Hanzo, "Visible light communications in heterogeneous networks: Paving the way for user-centric design," IEEE Wireless Commun., vol. 22, no. 2, pp. 8-16, 2015.

[171] X. Li, F. Jin, R. Zhang, J. Wang, Z. Xu, and L. Hanzo, "Users first: User-centric cluster formation for interference-mitigation in visiblelight networks," IEEE Trans. Wireless Commun., vol. 15, no. 1, pp. 39-53, 2016.

[172] X. Li, Y. Huo, R. Zhang, and L. Hanzo, "User-centric visible light communications for energy-efficient scalable video streaming," IEEE Trans. Green Commun. Netw., vol. 1, no. 1, pp. 59-73, 2017.

[173] M. Obeed, A. M. Salhab, S. A. Zummo, and M.-S. Alouini, "Joint power allocation and cell formation for energy-efficient VLC networks," in Proc. IEEE Int. Conf. Commun. (ICC), Kansas, USA, 2018.

[174] Z. Ding, X. Lei, G. K. Karagiannidis, R. Schober, J. Yuan, and V. K. Bhargava, "A survey on non-orthogonal multiple access for $5 \mathrm{G}$ networks: Research challenges and future trends," IEEE J. Sel. Areas Commun., vol. 35, no. 10, pp. 2181-2195, 2017.

[175] Y. Liu, Z. Qin, M. Elkashlan, Z. Ding, A. Nallanathan, and L. Hanzo, "Nonorthogonal multiple access for 5G and beyond," Proc. IEEE, vol. 105, no. 12, pp. 2347-2381, 2017.

[176] H. Marshoud, V. M. Kapinas, G. K. Karagiannidis, and S. Muhaidat, "Non-orthogonal multiple access for visible light communications," IEEE Photon. Technol. Lett, vol. 28, no. 1, pp. 51-54, 2016.

[177] L. Yin, W. O. Popoola, X. Wu, and H. Haas, "Performance evaluation of non-orthogonal multiple access in visible light communication," IEEE Trans. Commun., vol. 64, no. 12, pp. 5162-5175, 2016.

[178] Z. Yang, W. Xu, and Y. Li, "Fair non-orthogonal multiple access for visible light communication downlinks," IEEE Wireless Commun. Lett., vol. 6, no. 1, pp. 66-69, 2017.

[179] X. Zhang, Q. Gao, C. Gong, and Z. Xu, "User grouping and power allocation for NOMA visible light communication multi-cell networks," IEEE Commun. Lett., vol. 21, no. 4, pp. 777-780, 2017.

[180] Y. Yapici and I. Guvenc, "Non-orthogonal multiple access for mobile VLC networks with random receiver orientation," arXiv preprint arXiv:1801.04888, 2018 
[181] Z. Ding, Z. Yang, P. Fan, and H. V. Poor, "On the performance of nonorthogonal multiple access in $5 \mathrm{G}$ systems with randomly deployed users," IEEE Signal Process. Lett., vol. 21, no. 12, pp. 1501-1505, 2014.

[182] R. C. Kizilirmak, C. R. Rowell, and M. Uysal, "Non-orthogonal multiple access (NOMA) for indoor visible light communications," in 4th Int. Workshop Opt. Wireless Commun. (IWOW). IEEE, 2015, pp. $98-101$.

[183] B. Lin, X. Tang, Z. Ghassemlooy, C. Lin, M. Zhang, Z. Zhou, Y. Wu, and $\mathrm{H}$. $\mathrm{Li}$, "A NOMA scheme for visible light communications using a single carrier transmission," in First South American Colloq. Visible Light Communications (SACVLC). IEEE, 2017, pp. 1-4.

[184] H. Shen, Y. Wu, W. Xu, and C. Zhao, "Optimal power allocation for downlink two-user non-orthogonal multiple access in visible light communication," J. of Commun. and Inf. Netw., vol. 2, no. 4, pp. 5764, 2017.

[185] L. Yin, X. Wu, and H. Haas, "On the performance of non-orthogonal multiple access in visible light communication," in Proc. 26th Annu. Int. Symp. Pers. Indoor, and Mobile Radio Commun. (PIMRC). IEEE, 2015, pp. 1354-1359.

[186] B. Lin, Z. Ghassemlooy, X. Tang, Y. Li, and M. Zhang, "Experimental demonstration of optical MIMO NOMA-VLC with single carrier transmission," Opt. Commun., vol. 402, pp. 52-55, 2017.

[187] C. Chen, W.-D. Zhong, H. Yang, and P. Du, "On the performance of MIMO-NOMA based visible light communication systems," IEEE Photon. Technol. Lett., 2017.

[188] X. Guan, Q. Yang, Y. Hong, and C. C.-K. Chan, "Non-orthogonal multiple access with phase pre-distortion in visible light communication," Opt. Express, vol. 24, no. 22, pp. 25 816-25 823, 2016.

[189] X. Guan, Q. Yang, and C.-K. Chan, "Joint detection of visible light communication signals under non-orthogonal multiple access," IEEE Photon. Technol. Lett., vol. 29, no. 4, pp. 377-380, 2017.

[190] T. D. P. Perera, D. N. K. Jayakody, S. K. Sharma, S. Chatzinotas, and J. Li, "Simultaneous wireless information and power transfer (SWIPT): Recent advances and future challenges," IEEE Commun. Surveys Tuts., 2017.

[191] X. Lu, P. Wang, D. Niyato, D. I. Kim, and Z. Han, "Wireless networks with RF energy harvesting: A contemporary survey," IEEE Commun. Surveys Tuts., vol. 17, no. 2, pp. 757-789, 2015.

[192] R. King, D. Bhusari, D. Larrabee, X.-Q. Liu, E. Rehder, K. Edmondson, H. Cotal, R. Jones, J. Ermer, C. Fetzer et al., "Solar cell generations over $40 \%$ efficiency," Prog. Photovoltaics Res. Appl., vol. 20, no. 6, pp. 801-815, 2012.

[193] J. Fakidis, S. Videv, S. Kucera, H. Claussen, and H. Haas, "Indoor optical wireless power transfer to small cells at nighttime," J. Lightw. Technol., vol. 34, no. 13, pp. 3236-3258, 2016.

[194] T. Rakia, H.-C. Yang, F. Gebali, and M.-S. Alouini, "Optimal design of dual-hop VLC/RF communication system with energy harvesting," IEEE Commun. Lett., vol. 20, no. 10, pp. 1979-1982, 2016.

[195] — "Dual-hop VLC/RF transmission system with energy harvesting relay under delay constraint," in Globecom Workshops (GC Wkshps). IEEE, 2016, pp. 1-6.

[196] Y. Li, N. Huang, J. Wang, Z. Yang, and W. Xu, "Sum rate maximization for VLC systems with simultaneous wireless information and power transfer," IEEE Photonics Technol. Lett., vol. 29, no. 6, pp. 531-534, 2017.

[197] A. M. Abdelhady, O. Amin, A. Chaaban, and M. S. Alouini, "Resource allocation for outdoor visible light communications with energy harvesting capabilities," in Proc. IEEE Globecom Workshops (GC Wkshps), Dec 2017, pp. 1-6.

[198] G. Pan, H. Lei, Z. Ding, and Q. Ni, "On 3-D hybrid VLC-RF systems with light energy harvesting and OMA scheme over RF links," 2017.

[199] P. D. Diamantoulakis, G. K. Karagiannidis, and Z. Ding, "Simultaneous lightwave information and power transfer (SLIPT)," IEEE Trans. Green Commun. and Netw., 2018.

[200] H. G. Sandalidis, A. Vavoulas, T. A. Tsiftsis, and N. Vaiopoulos, "Illumination, data transmission, and energy harvesting: the threefold advantage of VLC," Appl. Opt., vol. 56, no. 12, pp. 3421-3427, 2017.

[201] M. Obeed, H. Dahrouj, A. M. Salhab, S. A. Zummo, and M.-S. Alouini, "DC-Bias allocation in cooperative VLC networks via joint information and energy transfer," in Proc. IEEE Global Commun. Conf. (Globecom), Abu Dhabi, 2018.

[202] — - "DC-Bias and power allocation in cooperative VLC networks for joint information and energy transfer," arXiv preprint arXiv:1812.11791, 2018.

[203] C. Carvalho and N. Paulino, "On the feasibility of indoor light energy harvesting for wireless sensor networks," Procedia Technol., vol. 17, pp. 343-350, 2014.

[204] J. L. Massey, "An introduction to contemporary cryptology," Proc. IEEE, vol. 76, no. 5, pp. 533-549, 1988.

[205] X. Zhou, Y. Zhang, and L. Song, Physical layer security in wireless communications. Crc Press, 2016.

[206] M. Obeed and W. Mesbah, "Efficient algorithms for physical layer security in two-way relay systems," Physical Communication, vol. 28, pp. $78-88,2018$.

[207] A. D. Wyner, "The wire-tap channel," Bell Labs Tech. J., vol. 54, no. 8, pp. 1355-1387, 1975.

[208] A. Mukherjee, S. A. A. Fakoorian, J. Huang, and A. L. Swindlehurst, "Principles of physical layer security in multiuser wireless networks: A survey," IEEE Commun. Surveys Tuts., vol. 16, no. 3, pp. 1550-1573, 2014

[209] X. Chen, D. W. K. Ng, W. H. Gerstacker, and H.-H. Chen, "A survey on multiple-antenna techniques for physical layer security," IEEE Commun. Surveys Tuts., vol. 19, no. 2, pp. 1027-1053, 2017.

[210] A. Mostafa and L. Lampe, "Enhancing the security of VLC links: Physical-layer approaches," in Summer Topicals Meeting Series (SUM). IEEE, 2015, pp. 39-40.

[211] G. J. Blinowski, "Practical aspects of physical and MAC layer security in visible light communication systems," Int. J. Electron. and Telecommun., vol. 62, no. 1, pp. 7-13, 2016.

[212] A. Mostafa and L. Lampe, "Physical-layer security for indoor visible light communications," in Proc. Int. Conf. Commun. (ICC). IEEE, 2014, pp. 3342-3347.

[213] C.-W. Chow, Y. Liu, C.-H. Yeh, C.-Y. Chen, C.-N. Lin, and D.-Z. Hsu, "Secure communication zone for white-light led visible light communication," Opt. Commun., vol. 344, pp. 81-85, 2015.

[214] A. Mostafa and L. Lampe, "Optimal and robust beamforming for secure transmission in MISO visible-light communication links," IEEE Trans. Signal Process., vol. 64, no. 24, pp. 6501-6516, 2016.

[215] H. Shen, Y. Deng, W. Xu, and C. Zhao, "Secrecy-oriented transmitter optimization for visible light communication systems," IEEE Photon. J., vol. 8, no. 5, pp. 1-14, 2016.

[216] T. V. Pham and A. T. Pham, "On the secrecy sum-rate of MU-VLC broadcast systems with confidential messages," in 10th Int. Symp. Communication Systems, Networks and Digital Signal Processing (CSNDSP). IEEE, 2016, pp. 1-6.

[217] — "Secrecy sum-rate of multi-user MISO visible light communication systems with confidential messages," Optik-Int. J. Light and Electron. Opt., vol. 151, pp. 65-76, 2017.

[218] M. A. Arfaoui, A. Ghrayeb, and C. Assi, "Achievable secrecy sum-rate of the MISO VLC broadcast channel with confidential messages," in Proc. Global Commun. Conf. (Globecom). IEEE, 2017, pp. 1-6.

[219] G. Pan, J. Ye, and Z. Ding, "On secure VLC systems with spatially random terminals," IEEE Commun. Lett., vol. 21, no. 3, pp. 492-495, 2017

[220] S. Cho, G. Chen, and J. P. Coon, "Secrecy analysis in visible light communication systems with randomly located eavesdroppers," in Int. Conf. Commun. Workshops (ICC Workshops). IEEE, 2017, pp. 475480.

[221] — - "Securing visible light communication systems by beamforming in the presence of randomly distributed eavesdroppers," IEEE Trans. Wireless Commun., vol. PP, no. 99, pp. 1-1, 2018.

[222] — "Physical layer security in visible light communication systems with randomly located colluding eavesdroppers," IEEE Wireless Commun. Lett., 2018.

[223] L. Yin and H. Haas, "Physical-layer security in multiuser visible light communication networks," IEEE J. Sel. Areas Commun., vol. 36, no. 1, pp. 162-174, 2018.

[224] X. Liu, X. Wei, L. Guo, Y. Liu, and Y. Zhou, "A new eavesdroppingresilient framework for indoor visible light communication," in Proc. Global Commun. Conf. (Globecom). IEEE, 2016, pp. 1-6.

[225] J. Classen, J. Chen, D. Steinmetzer, M. Hollick, and E. Knightly, "The spy next door: Eavesdropping on high throughput visible light communications," in Proc. 2nd Int. Workshop on Visible Light Commun. Syst. ACM, 2015, pp. 9-14.

[226] S. Cho, G. Chen, H. Chun, J. P. Coon, and D. Brien, "Impact of multipath reflections on secrecy in VLC systems with randomly located eavesdroppers," in Proc. Wireless Commun. and Netw. Conf. (WCNC). IEEE, apr 2018

[227] H. Le Minh, A. T. Pham, Z. Ghassemlooy, and A. Burton, "Secured communications-zone multiple input multiple output visible light communications," in Proc. Global Commun. Conf. (Globecom). IEEE, 2014, pp. 505-511. 
[228] M. A. Arfaoui, A. Ghrayeb, and C. Assi, "On the achievable secrecy rate of the MIMO VLC gaussian wiretap channel," in Proc. 28th Annu. Int. Symp. Pers. Indoor, and Mobile Radio Commun. (PIMRC). IEEE, 2017, pp. 1-5.

[229] Z. Chen and H. Haas, "Physical layer security for optical attocell networks," in Proc. Int. Conf. Commun. (ICC). IEEE, 2017, pp. 1-6.

[230] F. Wang, C. Liu, Q. Wang, J. Zhang, R. Zhang, L.-L. Yang, and L. Hanzo, "Secrecy analysis of generalized space-shift keying aided visible light communication," IEEE Access, 2018.

[231] M. F. Marzban, M. Kashef, M. Abdallah, and M. Khairy, "Beamforming and power allocation for physical-layer security in hybrid RF/VLC wireless networks," in 13th Int. Wireless Commun. and Mobile Computing Conf. (IWCMC). IEEE, 2017, pp. 258-263.

[232] G. Pan, J. Ye, and Z. Ding, "Secure hybrid VLC-RF systems with light energy harvesting," IEEE Trans. Commun., vol. 65, no. 10, pp. 4348-4359, 2017.

[233] - "Secrecy outage analysis of hybrid VLC-RF systems with light energy harvesting," in IEEE 18th Int. Workshop Signal Processing Advances in Wireless Commun. (SPAWC). IEEE, 2017, pp. 1-5.

[234] A. Mukherjee, "Secret-key agreement for security in multi-emitter visible light communication systems," IEEE Commun. Lett., vol. 20, no. 7, pp. 1361-1364, 2016.

[235] Y. M. Al-Moliki, M. T. Alresheedi, and Y. Al-Harthi, "Robust key generation from optical ofdm signal in indoor vlc networks," IEEE Photon. Technol. Lett., vol. 28, no. 22, pp. 2629-2632, 2016.

[236] - "Secret key generation protocol for optical OFDM systems in indoor VLC networks," IEEE Photon. J., vol. 9, no. 2, pp. 1-15, 2017.

[237] _ , "Physical-layer security against known/chosen plaintext attacks for OFDM-based VLC system," IEEE Commun. Lett., vol. 21, no. 12, pp. 2606-2609, 2017.

[238] H. Lu, L. Zhang, W. Chen, and Z. Wu, "Design and analysis of physical layer security based on ill-posed theory for optical OFDMbased VLC system over real-valued visible light channel," IEEE Photon. J., vol. 8, no. 6, pp. 1-19, 2016.

[239] Z. Wang and W. Qiu, "Secure image transmission over dft-precoded ofdm-vlc systems based on chebyshev chaos scrambling," Opt. Commun., vol. 397, pp. 84-90, 2017.

[240] Z. Wang, F. Chen, W. Qiu, S. Chen, and D. Ren, "A two layer chaotic encryption scheme of secure image transmission for DCT precoded OFDM-VLC transmission," Opt. Commun., vol. 410, pp. 94-101, 2018.

[241] S.-L. Shieh, C.-H. Lin, Y.-C. Huang, and C.-L. Wang, "On gray labeling for downlink non-orthogonal multiple access without SIC," IEEE Commun. Lett., vol. 20, no. 9, pp. 1721-1724, 2016.

[242] S.-L. Shieh and Y.-C. Huang, "A simple scheme for realizing the promised gains of downlink nonorthogonal multiple access," IEEE Trans. Commun., vol. 64, no. 4, pp. 1624-1635, 2016.

[243] Y. Liu, Z. Qin, M. Elkashlan, Y. Gao, and L. Hanzo, "Enhancing the physical layer security of non-orthogonal multiple access in large-scale networks." IEEE Trans. Wireless Communications, vol. 16, no. 3, pp. 1656-1672, 2017.

[244] Y. Zhang, H.-M. Wang, Q. Yang, and Z. Ding, "Secrecy sum rate maximization in non-orthogonal multiple access," IEEE Commun. Lett., vol. 20, no. 5, pp. 930-933, 2016.

[245] I. Lee, F. Kung, and M. Sim, "Performance enhancement of outdoor visible-light communication system using selective combining receiver," IET Optoelectronics, vol. 3, no. 1, pp. 30-39, feb 2009.

[246] M. S. Islim, S. Videv, M. Safari, E. Xie, J. J. D. McKendry, E. Gu, M. D. Dawson, and H. Haas, "The impact of solar irradiance on visible light communications," J. Lightw. Technol., vol. 36, no. 12, pp. 23762386, jun 2018.

[247] Y.-J. Zhu, Z.-G. Sun, J.-K. Zhang, and Y.-Y. Zhang, "A fast blind detection algorithm for outdoor visible light communications," IEEE Photon. J., vol. 7, no. 6, pp. 1-8, dec 2015.

[248] B. G. Guzman, V. P. G. Jimenez, M. C. Aguayo-Torres, H. Haas, and L. Hanzo, "Downlink performance of optical OFDM in outdoor visible light communication," IEEE Access, vol. 6, pp. 76 854-76866, 2018.

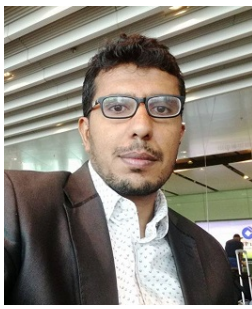

Mohanad Obeed (S'17) received the B.Eng. degree in computer and communication engineering from Taiz University, Taiz, Yemen, in 2008, the M.Sc. degree in electrical engineering from King Fahd University of Petroleum and Minerals (KFUPM), Dhahran, Saudi Arabia, in 2016.

$\mathrm{He}$ is currently pursuing the Ph.D. degree at KFUPM, Dhahran, Saudi Arabia. His research interests include visible light communications, cooperative communication, resource allocation, convex optimization, physical layer security, and energy

harvesting

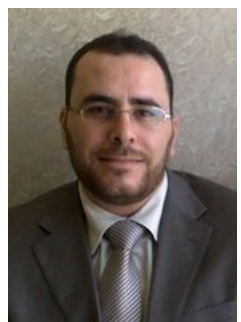

Anas M. Salhab (S'11-M'14-SM'17) received the B.Sc. degree in electrical engineering from Palestine Polytechnic University, Hebron, Palestine, in 2004, the M.Sc. degree in electrical engineering from Jordan University of Science and Technology, Irbid, Jordan, in 2007, and the Ph.D. degree from King Fahd University of Petroleum and Minerals (KFUPM), Dhahran, Saudi Arabia, in 2013. From 2013 to 2014, he was a Postdoctoral Fellow with the Electrical Engineering Department, KFUPM. He is currently an Assistant Professor and the Assistant Director of the Science and Technology Unit with the Deanship of Scientific Research, KFUPM. His research interest spans special topics in modeling and performance analysis of wireless communication systems, including cooperative relay networks, cognitive radio relay networks, free space optical networks, visible light communications, and co-channel interference. He was selected as an Exemplary Reviewer by the IEEE WIRELESS COMMUNICATIONS LETTERS for his reviewing service in 2014. Recently, he received the KFUPM Best Research Project Award as a Co-investigator among the projects in 2013/2014 and 2014/2015.

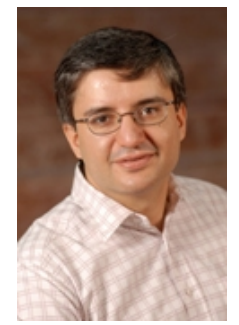

Mohamed-Slim Alouini (S'94, M'98, SM'03, F'09) was born in Tunis, Tunisia. He received the $\mathrm{Ph} . \mathrm{D}$. degree in Electrical Engineering from the California Institute of Technology (Caltech), Pasadena, CA, USA, in 1998. He served as a faculty member in the University of Minnesota, Minneapolis, MN, USA, then in the Texas A\&M University at Qatar, Education City, Doha, Qatar before joining King Abdullah University of Science and Technology (KAUST), Thuwal, Makkah Province, Saudi Arabia as a Professor of Electrical Engineering in 2009. His current research interests include the modeling, design, and performance analysis of wireless communication systems.

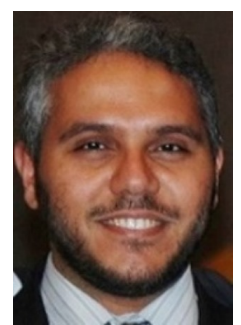

Salam A. Zummo (M'00-SM'08) received the B.Sc. and M.Sc. degrees in electrical engineering from the King Fahd University of Petroleum and Minerals (KFUPM), Dhahran, Saudi Arabia, in 1998 and 1999, respectively, and the Ph.D. degree from the University of Michigan, Ann Arbor, USA, in 2003. He is currently a Professor with the Electrical Engineering Department, KFUPM. Prof. Zummo has six issued U.S. patents and authored over 100 papers in reputable journals and conference proceedings. His research interests are in the area of wireless communications, including cooperative diversity, cognitive radio, multiuser diversity, scheduling, MIMO systems, error control coding, multihop networks, and interference modeling and analysis in wireless systems. He received the Saudi Ambassador Award for early Ph.D. completion in 2003, and the British Council/BAE Research Fellowship Awards in 2004 and 2006. He also received the KFUPM Excellence in Research Award from 2011 to 2012 